\title{
Bank Characteristics and the Interbank Money Market: A Distributional Approach
}

\author{
Giulia Iori, City University London \\ Burcu Kapar, City University London \\ Jose Olmo; University of Southampton
}

October 2014

\begin{abstract}
This paper studies the relationship between bank characteristics, such as size, nationality, operating currency and sovereign debt in the parent country, and the distribution of funding spreads observed in the e-MID interbank money market during the Great financial crisis. Our setup is a pseudo-panel with a random number of international banks acting in the interbank market in each period. We develop new econometric tools for panel data with random effects and discrete covariates, such as a nonparametric kernel estimator of the distribution function of the response variable conditional on a set of covariates and a consistent test of first order stochastic dominance. Our empirical results, based on these tests, shed light on the survivorship bias in the e-Mid market, and reveal the existence of a risk premium on small banks, banks with currencies different from the Euro, and banks based on countries under sovereign debt distress in the periphery of the European Union. Finally we assess the impact of policy intervention in the aftermath of the financial crisis.
\end{abstract}

Keywords: e-MID interbank money market, nonparametric kernel estimation, panel data methods, random effects, stochastic dominance tests.

JEL Classifications: C12, C14, G01, G20

\footnotetext{
${ }^{*}$ Corresponding address: School of Social Sciences, Economics Division, University of Southampton, Room 3015, Bld 58 (Murray Bld), Highfield Campus, Southampton, SO17 1BJ, UK. E-mail: J.B.Olmo@ soton.ac.uk. The research leading to this paper has received funding from the European Union, Seventh Framework Programme FP7/2007-2013 under grant agreement FOC "Forecasting Crisis", nr. 255987, and to a lesser extent from grant agreement CRISIS "Complexity Research Initiative for Systemic Instabilities", nr. 288501.
} 


\section{Introduction}

Interbank markets are the main instrument for the transmission of monetary policy targets from central banks to the overall economy. These markets are responsible for distributing liquidity across the financial system by allowing the transfer of funds from banks with a surplus to banks with a deficit. This is discussed for example in Ho and Saunders (1985), Bhattacharya and Gale (1987) and Freixas, Parigi and Rochet (2000), amongst others. These authors note the insurance role of interbank markets against idiosyncratic liquidity shocks.

At the same time, there is a wealth of theoretical papers analyzing the interbank money market and its role as accelerator of financial distress. Upper (2011) studies the occurrence of contagion in both liability and asset sides of banks' balance sheets. Other related articles are, on the liability side, Diamond and Dybvig (1983) modeling the fear of withdrawals, Acharya and Yorulmazer (2008) through information about asset quality or Acharya, Gromb and Yorulmazer (2008) via strategic behavior by potential lenders. On the asset side, Rochet and Tirole (1996) model contagion arising from interbank lending and Angelini, Mariesca and Russo (1996) discuss the role of disturbances in the payment system. Northcott (2002) studies the effect of security settlement, and Blavarg and Nimander (2002) the effect of FX settlement and derivative exposures. Cocco, Gomes and Martins (2009) concentrate on the effect of lending relationships on interbank market transactions.

In relation to the Global Financial Crisis of 2007-2008, the increase in counterparty risk and banks' liquidity hoarding behavior have been identified as important factors in the dry up of liquidity in interbank exchanges. Theoretical models by Freixas and Jorge (2008) and Heider, Hoerova, and Holthausen (2009) address the increase in counterparty risk as the main reason for the interbank market freeze. The study by Afonso, Kovnar and Schoar (2011) on the Fed Funds market provide empirical evidence to these models. Eisenschmidt and Tapking (2009), on the other hand, claim that liquidity hoarding behavior is the main reason for the market freeze during the crisis. Empirical examination of the UK market by Acharya and Merrouche (2013) and of the Fed Funds market by Ashcraft, McAndrews and Skeie (2011) support the liquidity hoarding theory. Increases in asymmetric information between banks during the crisis have also been postulated as possible causes of the breakdown in the interbank money market. This is theoretically studied by Freixas and Holthausen (2004) and empirically supported by Cassola, Holthausen and Duca (2010). These authors detect a shift from international money market activity to more domestic activity, leading to a segmentation of the European money market, with cross-border interbank trades executed mainly between banks with a high credit standing.

In addition to the dry up of liquidity, an increasing dispersion in the credit conditions of different banks has emerged in interbank markets during the financial crisis. There are several reasons to study the crosssection of lending and borrowing rates in the interbank money market. The spreads on these funding rates determine the level of risk aversion in the banking sector. Its cross-section provides useful information on the health of the banking system and alerts of the potential of systemic risk. This cross-section also sheds 
light on the existence of bank clubs facing similar borrowing conditions and can help to disentangle the reasons for the heterogeneity in such conditions. Banks operating in the interbank market differ in many dimensions such as customer profiles, level of competition, market power, investment portfolio strategies, credit risk exposure or existence of scale economies. The level of country-specific regulation also introduces heterogeneity in banks funding conditions due to cross-country differences in fees and commissions on financial transactions.

The aim of this study is to assess the effect of such bank characteristics on funding rates. We proxy these characteristics by a reduced set of easily available covariates such as bank nationality, size and operating currency, and control for the effect of sovereign debt in the parent country. Bank size captures differences in customer profile, business turnover, market power or the existence of scale economies. Nationality serves as a proxy for differences in government regulations and the level of domestic competition. This variable naturally captures differences in funding conditions between countries in the core and the periphery of the monetary union and also outside it. The operating currency is useful for capturing differences in banks performance derived from exchange rate fluctuations and the existence of different, if any, lenders of last resort. This variable can control for differences in risk exposure and leverage between banks inside and outside the monetary union. Bank nationality does not only affect interbank funding rates through different regulations but also through connections between the banking sector and the government sector in the form of credit lines, loans and sovereign bond purchases, that make banks' balance sheets very exposed to the performance of the overall domestic economy. These connections are not only limited to the reliance of the government sector on the banking sector. In recent years, countries in the periphery of the European Union (EU) have increased their debt levels to sustain the viability of their banking sector. These feedback effects have created strong links between banks and the public sector. The existence of a lender of last resort in some financial systems also helps shaping the characteristics of a bank by determining their credit risk exposure and leverage. The European interbank market is an interesting case because of the composition of their participants. Some of them operate in Euros and hence are under the umbrella of the European Central Bank (ECB), but there are others operating in a different currency but still trading in Euros on the interbank money market. All these characteristics determine the funding conditions obtained by banks in the interbank system that to a large extent are passed through to high street commercial banks.

The largest proportion of unsecured credit transactions takes place over-the-counter (OTC). Traders in OTC markets actively search for counterparties to a deal. When counterparties meet they negotiate terms privately, often ignoring prices available from other potential counterparties and with limited knowledge of trades recently negotiated elsewhere in the market. Data of the OTC trades can only be inferred from the payment system using algorithms such as the one in Furfine (1999). In Europe and USA, the e-MID market represents the only exception to OTC trading, by providing an electronic platform for Interbank Deposits. 'Buy' and 'sell' proposals are available on screens of the trading platform, along with the identity of the banks quoting them. Furthermore, information on the terms (prices and volumes) of executed trades are available to banks in real time. Another advantage of the e-MID is that interest rates reflect actual 
transactions, and therefore they are isolated from distortions impacting offered rates, such as Libor and Euribor. Finally, the heterogeneity in the characteristics of participating banks makes it preferable, for studies on funding conditions, to the Eonia market that only provides a weighted average of all uncollateralised overnight lending transactions in the interbank market in Euros undertaken by 35 panel banks.

Data availability, the lack of search friction and full transparency, have made the e-MID the subject of several studies in recent years. In particular, a number of studies have investigated how movements in borrowing costs depend on bank specific characteristics such as their size and creditworthiness, see Angelini, Nobili and Picillo (2011) and Gabrieli (2011a), on banks ability to exploit changing market microstructure conditions, see Gabbi et al. (2014), or they have concentrated on the intraday behaviour of the rates to gain further insights into the behaviour of banks and into the provision of liquidity in the overnight money market, see Baglioni and Monticini (2008), Baglioni and Monticini (2010), Angelini (2000), Beaupain and Durre (2013), Brunetti, Filippo and Harris (2011), Brousseau and Manzanares (2005). Some other studies analyze network topology of e-MID, see Iori and Precup (2007), Iori et al. (2007), Iori et al. (2008), Gabrieli (2011b) and Finger et al. (2012). Interestingly, most of these studies consider data up to the collapse of Lehman Brothers. Our empirical analysis extends these studies by incorporating data up to 2010. This choice of sample entails, however, a potential self-selection econometric problem due to the drop in the number of banks trading in the e-MID interbank money market after the collapse of Lehman Brothers. More specifically, it might be the case that after the occurrence of financial crises only banks with sound financial conditions would remain trading in the e-MID market whereas troubled banks would search for alternative ways of obtaining financing. Cassola, Holthausen and Duca (2010) find that, as a result of the turmoil, e-MID volumes decreased with respect to Eonia volumes, signalling European banks' preference to switch from a transparent electronic platform to an over-the-counter, more opaque, bilateral market. Nevertheless, according to Monticini and Ravazzolo (2011), e-MID was still representative for the whole Euro area money market. Although the share of non-Italian trading fell after the Lehman default, the e-MID remained anchored to the Euro area money market as witnessed by the low spread between the overnight interest rate traded in the e-MID and the Eonia.

Angelini, Nobili and Picillo (2011) address the potential self-selection problem in a similar study considering long term contracts in the e-MID market. These authors divide the sample into three different groups given by banks that entered the market after August 2007, those that stopped doing business on the market after August 2007 and those that were present throughout. Angelini, Nobili and Picillo (2011) conclude that distortions in the e-MID panel produced during the 2007 crisis period for long term funding rates do not influence their empirical findings. This conclusion is empowered by Arciero (2010) who finds that the e-MID data set is a good proxy for the transactions in the over-the-counter market both prior to and after August 2007. The findings of Beaupain and Durre (2011) are also in line with those of Arciero (2010) for the period between September 2000 and October 2007, including the beginning of the financial turbulence. A recent paper by Vento and Ganga (2010) studies whether the introduction of an anonymous and collateralized segment in the e-MID market, called the New MIC, improves liquidity distribution within 
the Euro area.

In contrast to most of the related literature we focus on modeling the distributional relationship between funding spreads and bank characteristics rather than the conditional mean of spreads. This is done to capture the existence of asymmetries in the distribution of spreads, heavy tails and multimodality. Funding rates are indicators of credit risk market expectations. They anticipate the occurrence of financial distress in the real economy by transferring credit risk in the form of large funding spreads from the banking sector to the real economy. Furthermore, the reaction of the spreads distribution to policy interventions provides an indication on the efficacy of the adopted measures. These empirical features highlight the importance of considering the entire distribution of cross-sectional spreads and not only mean and variance for drawing policy conclusions on the level of interest rates, the supply of money stock or assess the success of quantitative easing programs in passing through to the real economy. The presence of a right-skewed distribution with heavy tails is characteristic of an interbank money market with banks facing very heterogeneous funding rates. Similarly, a multimodal distribution reflects a cross-section comprising different clusters of banks. In order to properly capture these different empirical features we consider nonparametric kernel distribution estimation methods in a panel data setting. To control for the presence of heterogeneous effects in the conditional distribution of funding spreads we assume random effects in the unobserved component. This is a natural assumption given that the number of observations available in each period is different and the subjects in the sample can also vary from period to period. The proposed conditional analysis is determined by a vector of ordered and categorical regressors. We show that the discrete character of these variables entails very specific choices of the kernel function and also an asymptotic theory characterized by the absence of a bandwidth parameter in the rate of convergence of the standardized estimator. The statistical significance of the covariates is assessed by implementing least squares cross-validation methods, see Hall, Li and Racine (2004). This nonparametric methodology is in stark contrast with most of the related literature that explains the determinants of interbank funding rates using parametric panel data regression models, see Cocco, Gomes and Martins (2009), Afonso, Kovner and Schoar (2011) or Angelini, Nobili and Picillo (2011).

In order to control for the presence of structural breaks in the relationship between funding rates and bank characteristics we perform a separate econometric analysis over different subsamples covering periods before, during and after the 2007 and 2008 financial crises. Our paper proposes a similar strategy to Angelini, Nobili and Picillo (2011) to assess the existence and extent of self- selection in the sample of banks trading in e-MID with special emphasis around the recent crisis episodes. In contrast to the latter authors, our testing strategy to detect such survivorship bias consists of comparing the cross-sectional distribution of spreads for the group of surviving banks in the e-MID market with that for the group of banks that stopped trading in the e-MID market. The comparison between distributions is done by means of a stochastic dominance test of first order. The presence of random effects in the econometric model entails a serially correlated response variable and an extra term in the asymptotic distribution of the test statistic. To the best of our knowledge there are no tests of stochastic dominance that accommodate a panel of observations with 
random effects. One of the contributions of our study is then to extend these tests to this setting. We do this by developing the asymptotic theory of the appropriate test statistic and approximating such distribution by simulation and bootstrap methods. This testing framework is similar in spirit to Jun, Lee and Shin (2011) that develop stochastic dominance tests between potential outcomes in a panel treatment setup.

Our empirical findings reject an overwhelming presence of survivorship bias in our analysis across different subperiods. There is however some effect on both borrowing and lending segments during the early periods of the financial crisis. More specifically, the results of the stochastic dominance test show that the banks that dropped the e-MID market in the aftermath of the 2007 interbank crisis obtained in the preceding periods higher borrowing rates than those banks that remained in the market. However, we do not find statistically significant differences between distributions after the collapse of Lehman Brothers in September 2008. The rest of insights offered by the empirical analysis can be summarized as follows: Banks using the Euro as operating currency and in countries not affected by sovereign debt crises obtain lower funding costs. We also observe an asymmetric behavior between the borrowing and lending market; bank size is very relevant for explaining spreads in the borrowing market but not so important in the lending market. In particular we observe that borrowing spreads are a decreasing monotonic function of bank size but the evidence on a monotonic increasing relationship between lending spreads and size is not so overwhelming. Nevertheless, the empirical results reveal that the group of largest banks in the sample is able to set higher lending rates and enjoy lower borrowing rates. The nonparametric density estimator derived from our nonparametric methods uncovers the existence of bank clubs during and right after the collapse of Lehman Brothers. These differences, determined by heterogeneous funding restrictions across clubs, rapidly vanish in most cases suggesting the homogenization of the interbank money market following exceptional measures from central banks and governments across the world.

To summarise, our paper contributes to the literature by investigating the role of bank nationality, sovereign debt in the parent country, operating currency on spreads, calendar effects and monetary policy interventions on interbank funding rates; by the novel econometric technique used, and by expanding the analysis to data periods not covered in previous studies on this topic.

The remainder of this article is organized as follows. Section 2 explains the properties of the e-MID interbank market and describes our dataset. Section 3 describes the econometric methodology based on the application of nonparametric kernel methods and the statistical properties of the relevant nonparametric estimator. Section 4 extends the theory on stochastic dominance tests to a panel setting with random effects. Section 5 discusses the empirical findings obtained from applying these methods to our dataset. Lastly, Section 6 concludes. Proofs, tables and figures are collected in an appendix.

\section{Data and the e-MID Market}

This section introduces the characteristics of the e-MID interbank market and the dataset used for our empirical analysis. 


\subsection{The e-MID Market}

The e-MID company, established in 1990, makes use of an electronic platform to manage the interbank unsecured deposit market in Europe. It is the only electronic trading platform for interbank deposits in the Euro area and in the U.S. Under the supervision of Bank of Italy, credit institutions and investment companies can participate in this market if their total net asset size is respectively at least 10 million U.S. Dollars (or its equivalent in another currency) and 300 million euros (or its equivalent in another currency). Before the financial crisis the platform had 246 members from 29 EU countries and the U.S., of which thirty were central banks acting as market observers. Interbank deposit maturities range from overnight to one year with overnight contracts representing nearly $90 \%$ of total volume. After the crisis, the number of countries with banks actively participating in the e-MID market is sixteen: Austria, Belgium, Denmark, France, Germany, Greece, Ireland, Italy, Luxembourg, Netherlands, Norway, Poland, Portugal, Spain, Switzerland and United Kingdom.

One distinctive feature of the platform is that it is fully transparent. Buy and sell proposals appear on the platform with the identity of the bank posting them. In the overnight market segment a buy transaction is an interbank loan proposed by the borrowing bank submitting a bid quote on the screen, hence revealing itself as liquidity-short to the market; a sell transaction is an interbank loan initiated by the lender submitting an ask quote on the screen, hence revealing itself as liquidity-long. The platform does not offset any counterparty risk; search costs are identical for all platform participants. In this market each trader can choose to start the trade with any counterparty present in the book. The two parties can negotiate the terms and conditions of the specific trade, change the quantity/price or refuse the transaction at all.

\subsection{Data}

The dataset used for this study consists of all transactions recorded in the platform between 12 July 2006 and 8 September 2009. For each transaction we have comprehensive information about the date, time of trade, quantity exchanged, interest rate, transaction side (buy or sell) and the code of the quoting and ordering banks. Our database contains 125 Italian and 90 foreign banks acting as borrowers, lenders or both during the period of study. Although the identity of the banks is not available to us, we have information on capitalization for the Italian banks and the origin country for all banks. Italian banks are classified into five groups according to their weighted asset portfolio: major banks (higher than 60 billion euro), large banks (from 26 to 60 billion euro), medium banks (from 9 to 26 billion euro), small banks (from 1.3 to 9 billion euro) and minor banks (less than 1.3 billion euro). We only consider overnight $(\mathrm{O} / \mathrm{N})$ and the overnight long (ONL) contracts where ONL refers to contracts when more than one day is present between two consecutive business days. The interest rate is expressed as an annual rate and the amount of the transaction is quoted in millions of Euros. Table 1 presents average transaction size according to size and nationality. These statistics reveal the existence of correlation between transaction and institution size for Italian (domestic) banks. Transaction sizes are proportional to their asset size which is in line with the findings of Furfine 
(1999) for the FED funds market. Table 1 also reports the market share of foreign and different sized domestic banks with respect to the total number of transactions and amounts traded. Foreign banks have more than $50 \%$ total volume market share in both sides of the market. High participation of foreign banks in the e-MID market acknowledges its international character.

Table 1: Participation of Different Groups to the e-MID Market

\begin{tabular}{lcccccc}
\hline & Transaction Number & \multicolumn{2}{c}{ Total Volume } & \multicolumn{2}{c}{ Average Transaction Size } \\
\hline & Borrower & Lender & Borrower & Lender & Borrower & Lender \\
\hline Minor-Italian (31 Banks) & $5 \%$ & $10.4 \%$ & $2 \%$ & $3 \%$ & 17 & 16 \\
Small-Italian (63 Banks) & $47 \%$ & $50 \%$ & $16 \%$ & $19 \%$ & 17 & 19 \\
Medium-Italian (16 Banks) & $15 \%$ & $11 \%$ & $10 \%$ & $11 \%$ & 32 & 31 \\
Large-Italian (9 Banks) & $14 \%$ & $9 \%$ & $12 \%$ & $8 \%$ & 42 & 45 \\
Major-Italian (6 Banks) & $7 \%$ & $7 \%$ & $10 \%$ & $11 \%$ & 67 & 80 \\
Foreign Banks (90 Banks) & $11 \%$ & $11 \%$ & $50 \%$ & $51 \%$ & 220 & 212 \\
\hline
\end{tabular}

The trading activity of borrowers/lenders is affected by the reserve maintenance period announced by the ECB rather than by calendar month effects. This is so because banks need to comply with regulatory requirements on the amount of capital held over such periods. Empirically, for the Eonia rates, Gaspar, Quiros and Mendizabal (2008) report an increase in market activity towards the last days of the reserve maintenance period described by a remarkable increment in the number of transactions and the underlying volatility of interest rates. To capture these effects, we focus on monthly periods determined by the reserve maintenance period that correspond in our dataset to 38 monthly periods.

Our empirical analysis focuses on funding spreads rather than funding rates. The spread of each transaction is defined as the deviation of the transaction interest rate from the daily average market rate. More formally, let

$$
r x_{i j}=r_{i j}-\bar{r}_{j}
$$

with $r_{i j}$ an individual (borrowing or lending) interest rate outstanding for bank $i$ at day $j$ and $\bar{r}_{j}$ the average rate of all transactions in the interbank market on that day. In order to reduce the noise due to extreme movements on daily funding rates around the end of the reserve maintenance period we consider average monthly spreads computed over the entire reserve maintenance periods rather than daily observations. Thus for a bank $i$ executing $T_{i, t}$ transactions on a given reserve maintenance month $t$, the monthly average credit spread is

$$
y_{i t}=\frac{1}{T_{i, t}} \sum_{j=1}^{T_{i, t}} r x_{i j} .
$$

The number of borrowers and lenders in our sample varies from month to month between 62 and 127 and 78 and 156, respectively. The number of observations on a particular month may be smaller than the number of banks, simply because some banks may not be active during that period. This measure of spread generates a panel of observations in the cross-sectional dimension and serves us to assess the deviations of a particular 
bank funding conditions from the market average over each reserve maintenance period. This data structure closely corresponds to a pseudo-panel ${ }^{1}$. It is also worth noting that our definition of spread is robust to the presence of unobserved time-specific effects in the cross section of funding rates. Nevertheless, in order to control for the occurrence of structural breaks in the distributional relationship between funding rates and bank characteristics the empirical analysis is divided into six nonoverlapping subperiods.

Table 2 presents information about the subperiods considered in the study and determined by January 2007, August 2007, March 2008, September 2008 and March 2009. The first subsample, denoted as the 'Pre-crisis Period', covers the period July 2006 to January 2007 and is determined by the crash of the Shanghai Stock Exchange in February 2007, considered as one of the first signals of the financial crisis. The second subperiod is denominated as the 'Financial Markets Unease Period' and covers January 2007 to July 2007. The latter date closely corresponds with the start of the subprime mortgage crisis. The next period, denominated as 'Interbank Crisis Period', runs from July 07 to March 08. The collapse of Bear Sterns in March 2008 was the prelude to a period of increased tension in the investment banking sector reaching its peak on September 2008 with the collapse of Lehman Brothers, the knock-on effects on counterparty spreads and the subsequent freeze in lending activities between commercial banks. Thus the period between March 08 and September 08 is classified as the 'Pre-Lehman Period' and September 08 to March 09 as the 'Post-Lehman Period'. Lastly, we consider the period from March 09 to September 09 as the 'Post-Crisis Period' when the interbank market shows the first signals of recovery.

Table 2: Information about Sub-Periods

\begin{tabular}{llcc} 
Dates & Explanation & Number of Months & Periods \\
\hline July 06-Jan 07 & 2006-2 Before Crisis & 6 & Period 1 \\
Jan 07-July 07 & 2007-Financial Markets Unease & 6 & Period 2 \\
July 07-Mar 08 & Interbank Crisis & 8 & Period 3 \\
Mar 08-Sep 08 & Before Lehman Brothers Collapse & 6 & Period 4 \\
Sep 08-Mar 09 & After Lehman Brothers Collapse & 6 & Period 5 \\
Mar 09-Sep 09 & 2009-Post Crisis & 6 & Period 6 \\
\hline
\end{tabular}

The crisis episodes are characterized by a decline in market turnover and number of banks trading regularly in e-MID. This phenomenon can produce endogeneity problems due to self-selection issues. During periods of financial distress banks with funding constraints can prefer to trade over the counter instead of using the e-MID market and, thus, avoid giving signals of financial distress. The possibility of this survivorship bias needs to be taken into account and its effects empirically estimated when interpreting the results of the empirical analysis corresponding to the crisis periods. This is done in the empirical application.

\footnotetext{
${ }^{1} \mathrm{~A}$ pseudo-panel is an artificial panel based on a random sample of cross-sectional observations in each period, see Baltagi (2008, Chapter 10) for a detailed description.
} 


\section{Econometric Methodology}

This section is divided into three blocks. The first block presents a panel regression model relating the distribution of interbank funding spreads to a set of covariates. The second block discusses nonparametric kernel estimation of the conditional distribution using least squares cross-validation methods. The last block briefly reviews the theory on stochastic dominance tests and adapts it to the panel data context with random effects.

\subsection{Model}

In each period banks enter into the panel if they trade in the interbank market during that period. This feature implies that the number of observations in each period can vary and also that the individual subscript $i$ in the model below corresponds to a new and most likely different set of individuals in each period. These empirical features of our database suggest the choice of unobserved random effects to model the heterogeneity in the cross-section of funding spreads. More specifically, these unobserved effects, denoted by $\eta_{i}$, follow some distribution satisfying that $E\left[\eta_{i}\right]=0 \mathrm{and}^{2} V\left[\eta_{i}\right]=\sigma_{i}^{2}$, and are such that $\eta_{i}$ is independent of the error term and the vector of covariates for $i=1, \ldots, N$.

Let $d_{i t}=1\left(y_{i t} \leq y\right)$ with $1(\cdot)$ the indicator function taking a value of one if the argument is true and zero otherwise, and $y \in \Omega \subset \mathbb{R}$ with $\Omega$ a compact set, and let $I_{i t}$ be a $k$-dimensional vector of covariates with possible realizations denoted by a $k$-dimensional vector $x \in \Omega^{*} \subset \mathbb{R}^{k}$ a compact set; $N_{t}$ is the number of cross-sectional observations in each period and $t=1, \ldots, T$. We will also use interchangeably the alternative notation given by $N$ standing for the total number of banks in the system and $T_{i}$ for the number of active periods for bank $i$ with $i=1, \ldots, N$. For each $y$ fixed, consider the following panel regression model:

$$
d_{i t}(y)=\eta_{i}+g\left(I_{i t} ; y\right)+\varepsilon_{i t}(y), i=1, \ldots, N_{t} ; t=1, \ldots, T
$$

In the standard mean square error sense the function $g(x, y)$ obtained from considering $I_{i t}=x$ is interpreted as the conditional mean of $d_{i t}(y)$ given $I_{i t}=x$ that corresponds in this case to our object of interest: $F_{x}(y)=$ $P\left\{y_{i t} \leq y \mid I_{i t}=x\right\} ; \varepsilon_{i t}(y)$ is the stochastic error term that satisfies that $E\left[\varepsilon_{i t}(y) \mid I_{i t}\right]=0$ and $V\left[\varepsilon_{i t}(y) \mid I_{i t}\right]=\sigma^{2}(y)$ for all $y \in \Omega$. We further assume that $E\left[\varepsilon_{i s}(y) \varepsilon_{i t}(y) \mid I_{i s}, I_{i t}\right]=0$ for $s \neq t$.

In practice, the use of nonparametric regression techniques can be challenging if the conditioning sets are defined by a large number of covariates. Unfortunately, there is no easy solution to this problem that is intrinsic to the nonparametric regression literature. Partial solutions to mitigate the problem involve imposing some structure on the nonparametric regression, as for example an additive model, see Buja,

\footnotetext{
${ }^{2}$ The choice of a zero-mean random variable for modeling the random effects in the cross-section of data is without loss of generality given the definition of daily spread in (1). Thus if $\widetilde{\eta}_{i}$ is the random unobserved component corresponding to $r_{i t}$ in (1) with $\widetilde{\eta}_{i}$ satisfying that $E\left[\widetilde{\eta}_{i}\right]=\xi$ and $V\left[\widetilde{\eta}_{i}\right]=\widetilde{\sigma}_{i}^{2}$, then $r x_{i t}$ has, by construction, the following demeaned random effect $\widetilde{\eta}_{i}-\overline{\widetilde{\eta}}_{i}$

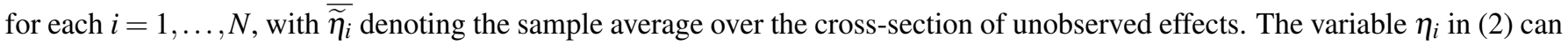
be interpreted as the monthly average random effect.
} 
Hastie and Tibshirani (1989), Hastie and Tibshirani (1989) and Newey (1994) for nonparametric estimation of additive models. We follow this approach and assume that the function $g\left(I_{i t} ; y\right)$ is separable with respect to each of the regressors in the covariate vector $I_{i t}=\left(I_{1, i t}, \ldots, I_{k, i t}\right)$. Thus the above equation can be written as

$$
d_{i t}(y)=\eta_{i}+\sum_{j=1}^{k} g_{j}\left(I_{j, i t} ; y\right)+\varepsilon_{i t}(y), i=1, \ldots, N_{t} ; t=1, \ldots, T,
$$

with $g_{1}\left(I_{1, i t} ; \cdot\right), \ldots, g_{k}\left(I_{k, i t} ; \cdot\right)$ a vector of real-valued functions describing the distribution function of the spreads conditional on each of the covariates. Note that the quantity of interest is $E\left[d_{i t}(y) \mid I_{i t}=x\right]$, however, as a byproduct, model (3) also allows us to identify the variance of the idiosyncratic random effects. These variance terms are defined as

$$
\sigma_{i}^{2}=E\left[\left(d_{i s}(y)-F_{x}(y)\right)\left(d_{i t}(y)-F_{x}(y)\right) \mid I_{i s}=x, I_{i t}=x\right], \text { for } s \neq t .
$$

\subsection{Nonparametric Estimation}

In this subsection we extend standard nonparametric estimators of the conditional distribution function of a random variable to a panel data framework defined by large $N$ and small $T$. The regression model (3) allows us to use a Nadaraya-Watson type kernel estimator, see Nadaraya (1965) and Watson (1964), to estimate the conditional distribution function conditional on $I_{i t}$.

Let $\widehat{F}_{x}(y)$ be the nonparametric estimator of $E\left[d_{i t}(y) \mid I_{i t}=x\right]$ and $n=\sum_{t=1}^{T} N_{t}=\sum_{i=1}^{N} T_{i}$. This estimator is constructed as

$$
\widehat{F}_{x}(y)=\frac{\frac{1}{n} \sum_{t=1}^{T} \sum_{i=1}^{N_{t}} d_{i t}(y) W_{h}\left(I_{i t} ; x\right)}{\widehat{\mu}(x)}
$$

where $\widehat{\mu}(x)=\frac{1}{n} \sum_{t=1}^{T} \sum_{i=1}^{N_{t}} W_{h}\left(I_{i t} ; x\right)$ is the kernel estimator of the multivariate density function $\mu(x)$ of the covariate vector $I_{i t} ; h=\left(h_{1}, \ldots, h_{k}\right)$ is a vector of smoothing parameters ${ }^{3}$, and $W_{h}\left(I_{i t} ; x\right)=\prod_{j=1}^{k} w_{h}\left(I_{j, i t} ; x_{j}\right)$. The choice of the kernel function $w_{h}\left(I_{j, i t} ; x_{j}\right)$ depends on whether the explanatory variable is continuous or discrete. Thus if the variable $I_{j, i t}$ is continuous the kernel function has the following location-scale representation $h^{-1} w\left(\frac{I_{j, i t}-x_{j}}{h}\right)$ with $w(\cdot)$ a univariate kernel function of the multiple choices existing in the literature, e.g. Gaussian, Uniform, Epanechnikov. The case of discrete explanatory variables requires separate attention. This is particularly relevant in our setting characterized by discrete covariates. More specifically, the factors relevant for our analysis of funding spreads can be further classified as ordered discrete factors, binary variables and multivariate binary discriminations of $\kappa$ mutually exclusive types. The first group is characterized by the variable bank size that consists of five ordered groups defined in terms of asset size; in

\footnotetext{
${ }^{3}$ For simplicity in the exposition, we hereafter use $h$ to denote all smoothing parameters attached to the covariates. Needless to say that in practice these choices differ across explanatory variables.
} 
the second group we will distinguish between Euro and Non-Euro countries and Crisis and Non-crisis areas, and in the last group of variables, we will consider European countries. Each type of regressor involves a different choice of kernel function $w_{h}\left(I_{j, i t} ; x_{j}\right)$. Unfortunately the convenient location-scale property assigned to the above kernel function is not available for the discrete case and is replaced by alternative kernel functions. Thus for the ordered discrete factor we use the kernel function proposed by Li and Racine (2008), defined as

$$
w_{h}\left(I_{j, i t} ; x_{j}\right)=h^{\left|x_{j}-z_{j}\right|}
$$

where $I_{j, i t}$ takes the value $z_{j} \in Z \subset \Omega^{*}$ with $Z$ the support of $I_{j, i t}$ defined by $\kappa$ possible realizations of the discrete ordered variable. For nominal discrete variables, we use the following variation of the kernel function developed by Aitchison and Aitken (1976) for binary covariates:

$$
w_{h}\left(I_{j, i t} ; x_{j}\right)=h^{1\left(\left|x_{j}-z_{j}\right|>0\right)}
$$

The extension of this kernel function to the case of $\kappa$ mutually exclusive binary types is

$$
w_{h}\left(I_{j, i t} ; x_{j}\right)=\left(\frac{h}{\kappa-1}\right)^{1\left(\left|x_{j}-z_{j}\right|>0\right)}
$$

In the three cases the smoothing parameter $h$ lies in the compact set $[0,1]$.

It is also worth discussing the role of the smoothing or bandwidth parameter. Nonparametric kernel estimation has been established as being relatively insensitive to the choice of the kernel function, the same cannot be said for bandwidth selection. There does not exist an automatic data-driven method for optimally selecting bandwidths when estimating a conditional distribution function in the sense that a weighted integrated mean square error is minimized. However, Hall, Li and Racine (2004) developed a data-driven method based on minimizing the integrated mean square error of the nonparametric kernel density estimate of a conditional density function when the conditioning variables are a mix of discrete and continuous variables. Given the close relationship between a conditional density function and a conditional distribution function, it makes sense to adopt the automatic data-driven selected bandwidths from minimizing the integrated mean square error of the kernel density estimator and use them for the estimation of the conditional distribution function. Thus, in the empirical application, we will use the least-squares cross validation function proposed in Hall, Li and Racine (2004) to choose the optimal bandwidth parameters. The advantage of this method over other alternatives, such as a rule of thumb or plug-in methods, is that cross-validation automatically discards irrelevant information from the conditioning vector. In our empirical exercise this method will allow us to determine which components of $I_{i t}$ are relevant and irrelevant, through assigning large smoothing parameters to the latter and consequently shrinking them toward the uniform distribution on the respective marginal distributions. 


\subsection{Statistical Properties}

The above estimator (5) inherits the properties of cross-sectional nonparametric kernel estimators of the conditional distribution function. The panel dimension adds observations to the estimates by pooling information from different periods. Following the mainstream literature on panel data we assume cross-sectional independence between the observations. Our data is however conditionally heteroscedastic due to the variance of the random effect $\eta_{i}$ that is allowed to vary across observations.

To derive the asymptotic distribution of (5) we assume that $I_{i t}$ only comprises discrete covariates. This assumption is highly relevant for deriving the appropriate convergence rate of the estimator; more specifically, the choice of discrete covariates allows (5) to achieve the parametric $N^{1 / 2}$ convergence rate instead of the nonparametric $\left(N h^{q}\right)^{1 / 2}$ rate obtained under the presence of $q$ continuous covariates. It trivially follows that the use of the time dimension in the panel also improves the speed of convergence of the estimator to achieve $(N T)^{1 / 2}$. Further assumptions on the model are:

A.1: The unobserved effects $\left\{\eta_{i}: i=1, \ldots, N\right\}$ are drawn from an unkown distribution function $F_{\eta}(\cdot)$ such that $E\left[\eta_{i}\right]=0$ and $V\left[\eta_{i}\right]=\sigma_{i}^{2}$ for $i=1, \ldots, N$. These random effects are independent across observations, independent of the error sequence $\varepsilon_{i t}(y)$ and independent of $\left\{I_{i t}: i=1, \ldots, N ; t=1, \ldots, T\right\}$.

A.2: The process $\left\{\left(y_{\cdot t}, I_{\cdot t}\right): t=1, \ldots, T\right\}$ is strictly stationary and absolutely regular with mixing coefficients $v(m)=O\left(m^{-A}\right)$ for some $A>v /(v-1)$. The process $\left\{\left(y_{i}, I_{i \cdot}\right): i=1, \ldots, N\right\}$ is independent and identically distributed over individuals.

A.3: The cumulative distribution function of $y_{i t}$ is uniformly continuous on $y \in \Omega$ and the function $\mu(x)$ is bounded away from zero for $x \in Z \subset \Omega^{*}$.

A.4: The sequence $\varepsilon_{i t}(y)$ satisfies that $E\left[\varepsilon_{i t}(y) \mid I_{i t}\right]=0$ for all $y \in \Omega$ and $E\left[\varepsilon_{i s}(y) \varepsilon_{i t}(y) \mid I_{i s}, I_{i t}\right]=0$ for $s \neq t$ and $i=1, \ldots, N$.

A.5: The kernel function $w_{h}\left(I_{j, i t} ; \cdot\right)$ is one of the following types: (6), (7) or (8), depending on the support of $I_{j, i t}$.

A.6: As $N \rightarrow \infty, h_{j} \rightarrow 0$ and $N^{1 / 2} h_{j} \rightarrow 0$ for $j=1, \ldots, k$, with $k$ the number of discrete regressors.

Assumption A.1 defines the random effect; A.2 imposes the stationarity of the process, the second part of the assumption is standard in the panel data literature and restricts the amount of cross-dependence. A.3 is a technical condition needed to extend the statistical properties of the estimator over $y \in \Omega$; A.4 defines the properties of the error term; A.5 determines the choice of kernel function for discrete covariates and A.6 is imposed to guarantee that the leading bias term of the nonparametric estimator (5) converges to zero. Interestingly, the panel dimension allows us to use bandwidth parameters that make the nonparametric estimator achieve convergence rates that can be considered optimal in the cross-sectional case. More specifically, Li and Racine (2008) show in a cross-sectional setting that $h=n_{c s}^{-1 / 2}$ with $n_{c s}$ the number of observations, is an optimal rate for the bandwidth parameter of a discrete covariate. In the cross-sectional case this rate of convergence does not remove the leading bias in the standarized version of (5), see proof of Theorem 
1. However, in our panel framework the choice $h=T / N$ satisfies A.6 and achieves the optimal rate of the cross-sectional case, e.g., for $N=100$ the optimal rate is obtained by considering $T=10$.

The following theorem formalizes the consistency and asymptotic normality of the estimator (5). For simplicity and without loss of generality, the following results consider a balanced panel with $n=N T$ observations. The extension to an unbalanced panel simply requires of some adjustments to the definition of $\lambda(x)$ in the asymptotic variance below.

Theorem 1.- Under assumptions A.1-A.6,

(i) $\widehat{F}_{x}(y)=F_{x}(y)+o_{P}(1)$ for all fixed $x \in \Omega^{*}$ and $y$ varying over its compact set $\Omega$.

(ii) $\widehat{F}_{x}(y)$ is such that

$$
n^{1 / 2}\left(\widehat{F}_{x}(y)-F_{x}(y)\right) \stackrel{d}{\rightarrow} N\left(0, \Sigma_{x}(y)\right),
$$

with $\Sigma_{x}(y)=F_{x}(y)\left(1-F_{x}(y)\right) / \mu(x)+E_{c}\left[\sigma_{i}^{2}\right](\mu(x)+\lambda(x)) / \mu^{2}(x)$, where $E_{c}[\cdot]$ is the cross-sectional expectation across individuals in the panel and $\lambda(x)=T^{-1} \sum_{\substack{s, t=1 \\ t \neq s}}^{T} P\left\{I_{i s}=x, I_{i t}=x\right\}$.

The following corollary presents the asymptotic distribution of the estimator under both strong persistence of the vector of covariates and serial independence. The first case corresponds to variables such as nationality or operating currency in our example, and the second case could reflect temporary characteristics. To further simplify the results we also assume that the observations are cross-sectionally homoscedastic.

\section{Corollary 1:}

(i) Let $I_{i t}$ be strongly persistent such that $P\left\{I_{i t}=x \mid I_{i s}=x\right\}=1$ with $s<t$, and $V\left[\eta_{i}\right]=\sigma^{2}$. The asymptotic result (9) holds with $\Sigma_{x}(y)=\left(F_{x}(y)\left(1-F_{x}(y)\right)+T \sigma^{2}\right) / \mu(x)$.

(ii) Let $I_{i t}$ be serially independent, that is, $P\left\{I_{i t}=x \mid I_{i s}=x\right\}=P\left\{I_{i t}=x\right\}$ with $s<t$, and $V\left[\eta_{i}\right]=\sigma^{2}$. The asymptotic result (9) holds with $\Sigma_{x}(y)=\left(F_{x}(y)\left(1-F_{x}(y)\right)+\sigma^{2}\right) / \mu(x)+(T-1) \sigma^{2}$.

It is worth noting the importance of the random effect in the asymptotic distribution of the estimator (5). Thus, depending on the time dimension of the panel, the contribution of the idiosyncratic variance can be several orders of magnitude larger than the contribution of the standard $F_{x}(y)\left(1-F_{x}(y)\right) / \mu(x)$ term obtained without allowing for random effects.

\section{Testing Framework}

Stochastic dominance provides a powerful methodology for comparing random variables beyond their expected value and variance. First order stochastic dominance compares the distribution function of the random variables of interest, the second order compares the expected value of the distributions, and so on. Fishburn (1977) showed the intrinsic relationship between stochastic dominance and mean-risk efficiency in a 
portfolio investment environment, and Davidson and Duclos (2000) used this concept to compare household income over a cross-section of different countries such as the U.S., Canada, the Netherlands, and Norway, for the year 1991. These authors suggested an approach based on tests of inequality constraints relying on a small number of comparisons. However, the fact that the comparisons are made at a fixed number of arbitrary chosen points is not a desirable feature, and introduces the possibility of test inconsistency. Barrett and Donald (2003) extend this method by proposing stochastic dominance tests based on comparison of the objects at all points in the support of income.

\subsection{Hypothesis Test}

The aim of these tests in the current framework is to allow us the distributional comparison between funding spreads conditional on the variables outlined above, namely, operating currency, nationality, existence of sovereign crisis and bank size. Our main interest is comparing the distribution of borrowing and lending rates for different values of the conditioning explanatory variables. Our panel data framework given by small $T$ and large $N$ entails a cross-sectional comparison between distribution functions in which the time dimension helps to increase the sample size by pooling observations across time within each subperiod. Hence, our approach is more similar in spirit to the cross-sectional methods developed in Davidson and Duclos (2000) and Barrett and Donald (2003) than to the dynamic tests of stochastic dominance recently proposed in Linton, Maasoumi and Whang (2005) and recently, Gonzalo and Olmo (2014), amongst others.

The testing framework of interest to compare funding spreads is

$$
H_{0}: F_{x}(y) \leq F_{\widetilde{x}}(y), \text { for all } y \in \Omega
$$

against

$$
H_{A}: F_{x}(y)>F_{\widetilde{x}}(y), \text { for some } y \in \Omega,
$$

with $x, \tilde{x}$ fixed values in the support of the random vector $I_{i t}$. This test can be interpreted as a test of stochastic dominance of first order between the distributions of funding spreads determined each by the value $x$ and $\widetilde{x}$ of the covariates.

The null hypothesis defined in (10) is composite, meaning that there are infinitely many conditions to be tested. Therefore, it is not clear in principle how one should derive the sampling distribution under the null hypothesis. Barrett and Donald (2003) in a cross-sectional setting and Linton, Maasoumi and Whang (2005) in a dynamic setting, focus on the least favorable case under the null hypothesis. This corresponds in our setting to assuming the equality between the distributions for every observation on the support of the compact set $\Omega$. The advantage of this approach resides in its simplicity for deriving the asymptotic theory of the test. However, the use of the least favorable case as a null hypothesis results in the largest critical values possible. This implies that the hypothesis that is really being tested is the equality of distributions under the null hypothesis. The no rejection of the null hypothesis (10) is a necessary condition 
for the presence of stochastic dominance of $F_{x}(\cdot)$ over $F_{\widetilde{x}}(\cdot)$, however, this test needs to be complemented with the reverse test characterized by swapping the roles of the random variables under both hypotheses $\left(H_{0}: F_{\widetilde{x}}(y) \leq F_{x}(y)\right.$ for all $y \in \Omega$ and $H_{A}: F_{\widetilde{x}}(y)>F_{x}(y)$ for some $\left.y \in \Omega\right)$. The rejection of the latter hypothesis implies that the distribution of the funding spreads conditional on $I_{i t}=x$ stochastically dominates its counterpart distribution conditional on $I_{i t}=\widetilde{x}$; otherwise the hypothesis of equality of distributions cannot be rejected. Finally, if both null hypotheses are rejected, there is statistical evidence to claim that the funding spreads corresponding to each conditioning setting are stochastically efficient of first order with the term efficient meaning absence of stochastic dominance of any of the distributions.

Let $S_{n}(y ; x, \widetilde{x})=n^{1 / 2}\left(\left(\widehat{F}_{x}(y)-F_{x}(y)\right)-\left(\widehat{F}_{\widetilde{x}}(y)-F_{\widetilde{x}}(y)\right)\right)$ with covariance function $K_{n}\left(y_{1}, y_{2} ; x, \widetilde{x}\right)$, and let $S_{\infty}(y ; x, \widetilde{x})$ denote a mean zero Gaussian process with covariance kernel $K_{\infty}\left(y_{1}, y_{2} ; x, \widetilde{x}\right) ; l^{\infty}(\Omega)$ denotes the functional space of all uniformly bounded real functions on $\Omega$ equipped with the sup-norm. To derive the weak convergence of the empirical process $S_{n}(y ; x, \widetilde{x})$ we need two further assumptions:

A.7: The sequence $\varepsilon_{i t}(y)$ is uniformly continuous on $y \in \Omega$ and $V\left[\varepsilon_{i t}(y) \mid I_{i t}\right]=\sigma^{2}(y)$ is Lipschitz continuous and bounded away from zero on their support.

A.8: $K_{n}\left(y_{1}, y_{2} ; x, \widetilde{x}\right)$ converges almost surely to $K_{\infty}\left(y_{1}, y_{2} ; x, \widetilde{x}\right)$, uniformly over $y_{1}, y_{2} \in \Omega$ for $x, \tilde{x} \in Z \subset \Omega^{*}$ fixed values.

Theorem 2.- Let $x, \widetilde{x}$ be fixed values in the support of $Z$. Under assumptions A.1-A.8,

$$
S_{n}(y ; x, \widetilde{x}) \stackrel{\omega}{\rightarrow} S_{\infty}(y ; x, \widetilde{x})
$$

with covariance kernel:

$$
\begin{aligned}
K_{\infty}\left(y_{1}, y_{2} ; x, \widetilde{x}\right) & =\left(F_{x}\left(\min \left(y_{1}, y_{2}\right)\right)-F_{x}\left(y_{1}\right) F_{x}\left(y_{2}\right)\right) / \mu^{2}(x)+\left(F_{\widetilde{x}}\left(\min \left(y_{1}, y_{2}\right)\right)-F_{\widetilde{x}}\left(y_{1}\right) F_{\widetilde{x}}\left(y_{2}\right)\right) / \mu^{2}(\widetilde{x}) \\
& +E_{c}\left[\sigma_{i}^{2}\right]\left(\mu^{2}(x)+\mu^{2}(\widetilde{x})+\lambda(x, \widetilde{x}) \mu(x) \mu(\widetilde{x})\right) /\left(\mu^{2}(x) \mu^{2}(\widetilde{x})\right)
\end{aligned}
$$

and $\lambda(x, \widetilde{x})=T^{-1} \sum_{\substack{s, t=1 \\ t \neq s}}^{T} P\left\{I_{i s}=x, I_{i t}=\widetilde{x}\right\} ; \omega$ denotes weak convergence in the $l^{\infty}(\Omega)$ space.

Under the least favourable version of the null hypothesis (10), the process $S_{n}(y ; x, \tilde{x})$ simplifies to $n^{1 / 2}\left(\widehat{F}_{x}(y)-\widehat{F}_{\widetilde{x}}(y)\right)$ and a natural Kolmogorov-Smirnov type statistic for testing $H_{0}$ is

$$
T_{n}(x, \widetilde{x})=n^{1 / 2} \sup _{y \in \Omega}\left|\widehat{F}_{x}(y)-\widehat{F}_{\widetilde{x}}(y)\right|
$$

Corollary 2.- Let $x, \tilde{x}$ be fixed values in the support of $Z$, and let $c_{\alpha}$ be the asymptotic critical value of the test at an $\alpha$ significance level. 
(i) Under $H_{0}$ and assumptions A.1-A.8,

$$
\lim _{N \rightarrow \infty} P\left\{T_{n}(x, \widetilde{x})>c_{\alpha}\right\} \leq \alpha
$$

with equality under the least favourable case.

(ii) Under $H_{A}$;

$$
\lim _{N \rightarrow \infty} P\left\{T_{n}(x, \tilde{x})>c_{\alpha}\right\}=1
$$

This result shows the consistency of the stochastic dominance test of first order for fixed values $x, \widetilde{x} \in$ $Z \subset \Omega^{*}$. Condition $(i)$ reveals a lack of power for null hypotheses more general than the least favourable case, see Barrett and Donald (2003), Linton, Maasoumi and Whang (2005) and related references, for discussion of this issue in different settings. The extension of these results to the functional space $\Omega \times \Omega^{*}$ defined by letting $(x, \widetilde{x})$ vary over its compact space is beyond the scope of this paper, but under appropriate assumptions it can be derived by applying the results in Chernozhukov, Lee and Rosen (2012).

Theorem 2 shows that under the absence of random effects the Kolmogorov-Smirnov test converges in distribution to the supremum of a zero mean Gaussian processs with critical values that can be tabulated in some cases. The existence of random effects in the regression model (3) entails the presence of nuisance parameters in the asymptotic distribution, hence, critical values cannot be universally tabulated and we need to rely on simulation and bootstrap techniques. The following subsection discusses such approximations to the asymptotic null distribution of the test.

\subsection{Approximation of the Asymptotic P-Values}

There are several alternatives explored in the literature for testing stochastic dominance, namely, simulation and iid bootstrap methods as in Barrett and Donald (2003), subsampling and bootstrap as in Linton, Maasoumi and Whang (2005), block bootstrap for time series as in Scaillet and Topaloglou (2010), and recently, a multiplier method for nonparametric kernel regression as proposed in a different iid setting by Chernozhukov, Lee and Rosen (2012) and in a time series stochastic dominance setting by Gonzalo and Olmo (2014). In this subsection we explore these strategies adapted to our panel data framework. In particular, we discuss a bootstrap method and a simulation method in the spirit of the multiplier method proposed by van der Vaart and Wellner (1996) to approximate the null distribution of $T_{n}(x, \widetilde{x})$.

The cross-sectional independence of the observations suggests the use of naive bootstrap techniques. The availability of a panel structure for the data given by a small $T$ implies that the resampling method can exploit the time dimension without having to impose further restrictions on the extent of serial correlation in the data. A simple way of doing so is extracting iid observations using a naive nonparametric bootstrap; instead of obtaining single observations from each draw we suggest retaining the time structure of the panel

by stacking all the time series observations corresponding to that draw as a block given by $\left(y_{i t}^{*}, I_{i t}^{*}\right)$ for 
$t=1, \ldots, T_{i}$. This method combines the simplicity of the naive iid bootstrap with the potential of the block bootstrap to replicate serial dependence structures. This bootstrap methodology replicates the unbalanced panel structure of the original data. The bootstrap test statistic corresponding to this method is

$$
T_{n}^{*}(x, \widetilde{x})=\sqrt{n^{*}} \sup _{y \in \Omega}\left|\left(\widehat{F}_{x}^{*}(y)-\widehat{F}_{\widetilde{x}}^{*}(y)\right)\right|-T_{n}(x, \widetilde{x}),
$$

with

$$
\widehat{F}_{z}^{*}(y)=\frac{\frac{1}{n^{*}} \sum_{i=1}^{N} \sum_{t=1}^{T_{i}^{*}} d_{i t}^{*}(y) W_{h}\left(I_{i t}^{*} ; z\right)}{\widehat{\mu}^{*}(z)}, \text { for } z=x, \widetilde{z}
$$

where $\widehat{\mu}^{*}(z)=\frac{1}{n^{*}} \sum_{i=1}^{N} \sum_{t=1}^{T_{i}^{*}} W_{h}\left(I_{i t}^{*} ; z\right)$ is the kernel estimator of the bootstrap multivariate density function $\mu^{*}(z)$ of the covariate vector $I_{i t}^{*} ; n^{*}=\sum_{i=1}^{N} T_{i}^{*}$ with $T_{i}^{*}$ the block length corresponding to each bootstrap resampled observation.

The bootstrap distribution of the test statistic is approximated by obtaining $B$ independent replicas of $T_{n}^{*}(x, \widetilde{x})$. It is not difficult to see that conditional on the panel of observations available to the econometrician this bootstrap test is consistent under both null and alternative hypotheses. Mathematically, under assumptions A.1-A.8 and the null hypothesis $H_{0}$;

$$
\lim _{N \rightarrow \infty} P\left\{T_{n}(x, \widetilde{x})>c_{\alpha}^{*} \mid\left(y_{i t}, I_{i t}\right)\right\} \leq \alpha,
$$

with equality under the least favourable case, and under $H_{A}$;

$$
\lim _{N \rightarrow \infty} P\left\{T_{n}(x, \widetilde{x})>c_{\alpha}^{*} \mid\left(y_{i t}, I_{i t}\right)\right\}=1
$$

where $c_{\alpha}^{*}$ is the critical value obtained as the $(1-\alpha)$-quantile of the bootstrap distribution.

Alternatively, Hansen (1996) proposes an alternative to generate iid copies of the score of linear regression models that relies on a multiplier method, see van der Vaart and Wellner (1996). This author approximates the asymptotic distribution of a Wald type test by applying a Central Limit Theorem to the simulated score functions. This so called multiplier Central Limit Theorem can be immediately applied in our context. The idea is to introduce randomness into the existing test statistic by multiplying the individual outcomes by an iid random variable with finite support and mean zero. To simplify the description of the method we will assume, without loss of generality, that the panel is balanced and $n=N T$. More specifically, as sketched in the proof of Theorem 2 , the test statistic $T_{n}(x, \widetilde{x})$ can be expressed as

$$
T_{n}(x, \widetilde{x})=n^{1 / 2} \sup _{y \in \Omega}\left|\frac{1}{N} \sum_{i=1}^{N} \widehat{s_{i}}(y ; x, \widetilde{x})\right|,
$$


with $\widehat{s}_{i}(y ; x, \widetilde{x})=\frac{1}{T} \sum_{t=1}^{T} s_{i t}^{o}(y ; x, \widetilde{x})$ for $i=1, \ldots, N$ and $s_{i t}^{o}(y ; x, \widetilde{x})=d_{i t}(y) W_{h}\left(I_{i t} ; x\right) / \widehat{\mu}(x)-d_{i t}(y) W_{h}\left(I_{i t} ; \widetilde{x}\right) / \widehat{\mu}(\widetilde{x})$.

Let $u_{i}$ be an iid random variable with zero mean and finite support, and let $T_{n}^{*}(x, \widetilde{x})$ be the simulated counterpart test statistic generated by replacing $\widehat{s_{i}}(y ; x, \widetilde{x})$ by $\widehat{s_{i}}(y ; x, \widetilde{x}) u_{i}$ in the preceding expression. This simulated process is robust to the presence of random effects in the data and converges, under standard assumptions and conditional on the available panel data, to an independent replica of the asymptotic null distribution of the test derived in Corollary 2. In this way, this simulated test statistic provides a consistent test under both null and alternative hypotheses and the critical values can be approximated by generating $B$ independent simulated copies of the test statistic and retaining the empirical $(1-\alpha)$-quantile of the simulated distribution. The proof of this result is analogous to that of Theorem 2 in Hansen (1996).

\section{Empirical Findings}

In this section we implement the above methodology to explore the distributional relationship between funding spreads on the interbank market and bank characteristics. Table 3 reports the bandwidth parameters of the least squares cross-validation method obtained from the estimates of the density function of spreads conditional on our set of covariates. The reported values illustrate the statistical relevance of these covariates and are robust across subperiods highlighting the importance of our conditional analysis for describing the cross-section of borrowing and lending spreads.

Table 3: Optimal Bandwidth Parameters of Conditional Density Estimation of Spread on Nationality \& Asset Size

\begin{tabular}{|c|c|c|c|c|c|c|c|c|c|c|}
\hline & Period & $\begin{array}{c}\text { Euro- } \\
\text { NonEuro }\end{array}$ & Spread & Obs. No & $\begin{array}{l}\text { Crisis- } \\
\text { NonCrisis }\end{array}$ & Spread & Obs. No & $\begin{array}{l}\text { Asset } \\
\text { Size }\end{array}$ & Spread & Obs. No \\
\hline \multirow{6}{*}{ : } & Period 1 & $0.50(0.5)$ & 0.001 & 266 & $0.25(0.5)$ & 0.003 & 266 & $0.09(1.00)$ & 0.002 & 462 \\
\hline & Period 2 & $0.40(0.5)$ & 0.003 & 276 & $0.10(0.5)$ & 0.003 & 276 & $0.23(1.00)$ & 0.003 & 441 \\
\hline & Period 3 & $0.50(0.5)$ & 0.007 & 307 & $0.25(0.5)$ & 0.006 & 307 & $0.17(1.00)$ & 0.007 & 582 \\
\hline & Period 4 & $0.25(0.5)$ & 0.014 & 227 & $0.01(0.5)$ & 0.004 & 227 & $0.27(1.00)$ & 0.012 & 411 \\
\hline & Period 5 & $0.37(0.5)$ & 0.036 & 135 & $0.01(0.5)$ & 0.036 & 135 & $0.07(1.00)$ & 0.036 & 374 \\
\hline & Period 6 & $0.50(0.5)$ & 0.018 & 116 & $0.02(0.5)$ & 0.014 & 116 & $0.07(1.00)$ & 0.018 & 365 \\
\hline \multirow{6}{*}{ لِّ } & Period 1 & $0.14(0.5)$ & 0.002 & 303 & $0.05(0.5)$ & 0.002 & 303 & $0.26(1.00)$ & 0.002 & 605 \\
\hline & Period 2 & $0.25(0.5)$ & 0.002 & 344 & $0.10(0.5)$ & 0.002 & 344 & $0.26(1.00)$ & 0.003 & 571 \\
\hline & Period 3 & $0.25(0.5)$ & 0.006 & 434 & $0.09(0.5)$ & 0.007 & 434 & $0.66(1.00)$ & 0.004 & 741 \\
\hline & Period 4 & $0.02(0.5)$ & 0.011 & 297 & $0.25(0.5)$ & 0.011 & 297 & $0.19(1.00)$ & 0.008 & 553 \\
\hline & Period 5 & $0.50(0.5)$ & 0.038 & 176 & $0.50(0.5)$ & 0.038 & 176 & $0.31(1.00)$ & 0.023 & 510 \\
\hline & Period 6 & $0.50(0.5)$ & 0.026 & 117 & $0.50(0.5)$ & 0.026 & 117 & $0.13(1.00)$ & 0.014 & 499 \\
\hline
\end{tabular}

Note: Bandwidth Parameters of Conditional Densities are estimated with Least Squares Cross-Validation Method for each subperiods covering from July 2006 to August 2009. Kernel Function for Spread is Gaussian and Kernel Functions for Euro/NonEuro and Crisis/Non-Crisis classifications are Aitchison and Aitken(1976).

Before proceeding with the empirical analysis we clarify several points. First, for simplicity and sake of presentation, the estimation of (5) in each of the exercises below has been done using the explanatory 
variable of interest as a single regressor. Under the assumption that the regressor variables are mutually uncorrelated this assumption introduces no bias in the estimate of $F_{x}(y)$ and also yields a consistent estimator of the relevant quantity for all $y \in \Omega$. The suitability of this assumption is confirmed empirically by the correlations between the set of regressors. More specifically, the correlation between nationality and currency and nationality and sovereign debt are 0.14 and 0.29 , respectively. The correlation between currency and sovereign debt is 0.92 . Unfortunately, our sample only contains data on size for Italian banks, hence it is not possible to compute the correlation between this variable and the rest of regressors. These results suggest that the correlations between nationality and currency and nationality and sovereign debt are quite low, validating the above assumption. The correlation between sovereign debt and currency is, on the other hand, close to one. Note however that this correlation is somehow misleading due to the lack of variability between the variables. More specifically, all of the countries in our analysis under sovereign distress belong to the Euro. This strong correlation does not invalidate our analysis. On the contrary, the analysis of the distribution of funding rates conditional on sovereign debt can be interpreted as a particular case of the analysis conditional on the Euro currency but also conditional on those countries from the Euro area under sovereign distress. In these cases it is standard practice in parametric econometric models to remove one of the regressors to avoid multicollinearity issues. This is the motivation for only including one single regressor in the analyses of currency and sovereign debt reported below.

Second, to illustrate better the relationship between spreads for different values of the covariates and over time we report kernel density estimates rather than their distributional counterparts estimated in (5). This change of estimator introduces an extra kernel density function for the continuous random variable $y_{i t}$ that is chosen in our empirical analysis to be Gaussian.

\subsection{Self-selection Issues}

Self-selection arises in situations in which individuals select themselves into a group. In this setting, the group is given by those banks that trade in the interbank e-MID market. Under the assumption that sample selection into this group is randomized, that is, sample selection is independent of all other observable and unobservable factors, there is no bias in the model parameter estimates and sample selection is thus exogenous. However, if the variables determining the selection into the e-MID sample are not captured by our set of explanatory variables but are correlated with them and the funding spreads, then, sample selection produces a statistical bias in the parameter estimates. In our specific setting, sample selection bias can arise during periods of financial distress if the group of banks trading in the e-MID market correspond to banks with sound financial conditions. This phenomenon is related to the existence of survivorship bias in the hedge funds literature. In a parametric setting, this survivorship bias can be corrected by the inclusion of control variables determining sample selection. This choice is, however, empirically difficult in our setting given the unavailability of reliable variables proxying financial conditions. Instead, we propose to assess the existence and extent of this self-selection issue by comparing, for each period, the distribution of funding 
spreads between the banks that remain actively trading in the e-MID and those banks that stop trading in the e-MID in that specific period. By doing so, we hope to determine if sample selection is random or at least randomized in each period, or instead, is determined by the history of funding spreads proxying financial conditions. In the latter case our estimates of the distribution function of funding spreads obtained from the e-MID sample would be biased conservative estimates of the distribution of the population of banks' funding spreads.

Table 4 reports the p-value of the relevant stochastic dominance tests. In order to obtain some insight into the dynamics of the distributions for each of the two groups discussed above we repeat the test for all the periods before the specific episode under study. The results do no shed overwhelming evidence on the existence of self selection by surviving banks. This is particularly relevant for the period around the collapse of Lehman Brothers. The decline in the number of banks trading in the e-MID market after this event cannot be attributed to a survivorship bias. More specifically, banks that stopped trading in the e-MID during this period did not consistently receive higher borrowing rates than those banks that remained openly trading after the collapse of Lehman Brothers. This result is surprisingly reversed for the periods around the interbank crisis period and the pre-Lehman period. Table 4 reveals some survivorship bias during these periods suggesting that banks that stopped trading in the e-MID market were targeted as troubled banks and consistently received statistically significant higher borrowing rates than the rest of banks still active after these periods of financial unease. Nevertheless, the results in Table 4 reveal the existence of some survivorship bias on the lender segment for Periods 5 and 6. More specifically, the stochastic dominance test indicates that banks that remain active in the e-MID market in these periods exhibited higher lending rates in Periods 4 and 5 than those banks that subsequently dropped from the sample.

Overall our analysis does not support models such as Heider, Hoerova, and Holthausen (2009), which attributes higher interbank rates to adverse selection, with the more creditworthy participants leaving the market. Neither we find overwhelming evidence that a stigma effect associated to the demand for interbank deposits has led bad borrowers to prefer anonymous markets over transparent ones. While the decrease in volume in the e-MID could be the result of a shift from unsecured to the secured market, or from a transparent to an opaque market, the empirical evidence shed by the stochastic dominance tests is favorable to the hypothesis of random sample selection for most of the subperiods under study. The interpretation of the results for the period around the interbank crisis should be taken with caution, though. During this period the results in Table 4 confirm the existence of some survivorship bias and suggest that the p-values of the stochastic dominance tests are a conservative measure of the $\mathrm{p}$-values of the tests using distribution function estimates obtained from a random sample of banks. It is worth noting that even during this period we find statistical evidence to reject the relevant null hypotheses corresponding to the different exercises below. 
Table 4: Stochastic Dominance Test Results for Active and Drop Banks

\begin{tabular}{|c|c|c|c|c|c|}
\hline \multirow[b]{2}{*}{ Drop (Period) } & \multirow[b]{2}{*}{ Period(Analysed) } & \multicolumn{2}{|c|}{ Borrower } & \multicolumn{2}{|c|}{ Lender } \\
\hline & & $\begin{array}{c}H_{0}: F_{\text {Active }}(x) \leq \\
F_{\text {Drop }}(x)\end{array}$ & $\begin{array}{c}H_{0}: F_{\text {Drop }}(x) \leq \\
F_{\text {Active }}(x)\end{array}$ & $\begin{array}{c}H_{0}: F_{\text {Active }}(x) \leq \\
F_{\text {Drop }}(x)\end{array}$ & $\begin{array}{c}H_{0}: F_{\text {Drop }}(x) \leq \\
F_{\text {Active }}(x)\end{array}$ \\
\hline Drop $_{\text {Period2 }}$ & Period 1 & 0.36 & 0.12 & 0.87 & 0.03 \\
\hline \multirow{2}{*}{ Drop $_{\text {Period3 }}$} & Period 1 & 0.003 & 0.97 & 0.99 & 0.12 \\
\hline & Period 2 & 0.01 & 0.65 & 0.46 & 0.32 \\
\hline \multirow{3}{*}{ Drop $_{\text {Period4 }}$} & Period 1 & 0.01 & 0.59 & 0.99 & 0.01 \\
\hline & Period 2 & 0.001 & 0.72 & 0.81 & 0.18 \\
\hline & Period 3 & 0.43 & 0.58 & 0.002 & 0.96 \\
\hline \multirow{4}{*}{ Drop $_{\text {Period5 }}$} & Period 1 & 0.68 & 0.28 & 0.01 & 0.79 \\
\hline & Period 2 & 0.30 & 0.54 & 0.001 & 0.85 \\
\hline & Period 3 & 0.16 & 0.55 & 0.00 & 0.78 \\
\hline & Period 4 & 0.15 & 0.93 & 0.00 & 0.92 \\
\hline \multirow{5}{*}{ Drop ${ }_{\text {Period6 }}$} & Period 1 & 0.25 & 0.51 & 0.31 & 0.71 \\
\hline & Period 2 & 0.007 & 0.58 & 0.0004 & 0.94 \\
\hline & Period 3 & 0.0008 & 0.64 & 0.00 & 0.90 \\
\hline & Period 4 & 0.26 & 0.47 & 0.04 & 0.89 \\
\hline & Period 5 & 0.79 & 0.01 & 0.68 & 0.24 \\
\hline
\end{tabular}

Note: Active means banks have transactions in all periods as well as till the last month in the sixth period. Drop means banks have no transaction in the specified period. Drop (Period) indicates in which period banks has stopped trading. $\mathrm{x}$ represents spread level. The table reports empirical p-values of stochastic dominance test.The period under study is from 12 July 2006 to 8 September 2009.

\subsection{Size Effects}

Asset size is an important variable to determine the characteristics of commercial banks. In fact, Angelini, Nobili and Picillo (2011) note that before the crisis bank asset size is the only relevant variable that determines borrowing spreads. These authors also consider the rating and the bank capitalization ratio as potential proxies for bank characteristics, however, they find that these variables are not statistically significant. This empirical finding is supported for the U.S. money market by early studies such as Stigum (1990), Allen and Saunders (1986) and Furfine (2001). These articles draw attention to the tiering structure in the federal funds market by which large institutions get favourable rates compared to smaller institutions regardless the side of the transaction they are on.

Our dataset on the e-MID market only contains information on asset size for Italian banks but not for the rest of nationalities. There are 125 Italian banks in our sample. Strictly speaking, this implies that our analysis is conditional on asset size and Italian nationality of at least one of the two counterparties. We believe, though, that the conclusions of this analysis can be extrapolated to assess the impact of size structure on funding rates for banks operating in any other EU country. The conditioning information set is defined by a discrete ordered random variable taking five possible values where a value of one corresponds to those banks with smallest capitalization, denoted as minor banks and a value of five corresponds to the largest banks, denoted as major banks. In between, there are small banks, medium banks, and large banks.

Figures 1 and 2 report the nonparametric densities for the borrowing and lending spreads, respectively, over each subperiod. Table 5 reports the stochastic dominance tests for all pairwise combinations of banks 
in terms of asset size. The results of the tests clearly show that borrowing rates are a monotonic decreasing function of asset size. The study of the lending spreads also reveals a statistical significant effect of size on the cross-sectional distribution of spreads. In this case major banks dominate stochastically the rest of banks. This result is strengthened after the collapse of Lehman Brothers and highlights the shield against risk aversion offered by asset size in periods of financial distress. Banks take advantage of their size to obtain lower borrowing rates from the interbank market and offer at the same time higher lending rates. These empirical findings are strongly related to too big to fail theories. While our investigation confirms the results of Gabrieli(2011a), the analysis of the distributions provides additional insights. These charts describe an interbank money market that abruptly moves from a stable market condition defined by similar funding rates across banks of different size to a disrupted market at the start of the crisis. The density functions of the cross-section of borrowing spreads uncover important differences between the groups of banks. This difference is accentuated after the tensions in debt markets that emerged in September 2008. The distributions generally become broader over time for all groups, with banks in the left tail of the distribution enjoying significantly smaller borrowing rates than the rest of banks. Overall larger banks experience better rates, but within each group we find groups of banks performing considerably better or worse than the market average. The nonparametric density estimates also reveal modes in the distribution of borrowing spreads. These modes capture the existence of bank clubs, possibly characterised by their degree of creditworthiness. After the crisis the differences in banks' borrowing conditions in terms of asset size decrease but are still noticeable. The analysis of the lending segment in Figure 2 exhibits less contrast. Lending rates are similar across bank sizes. 


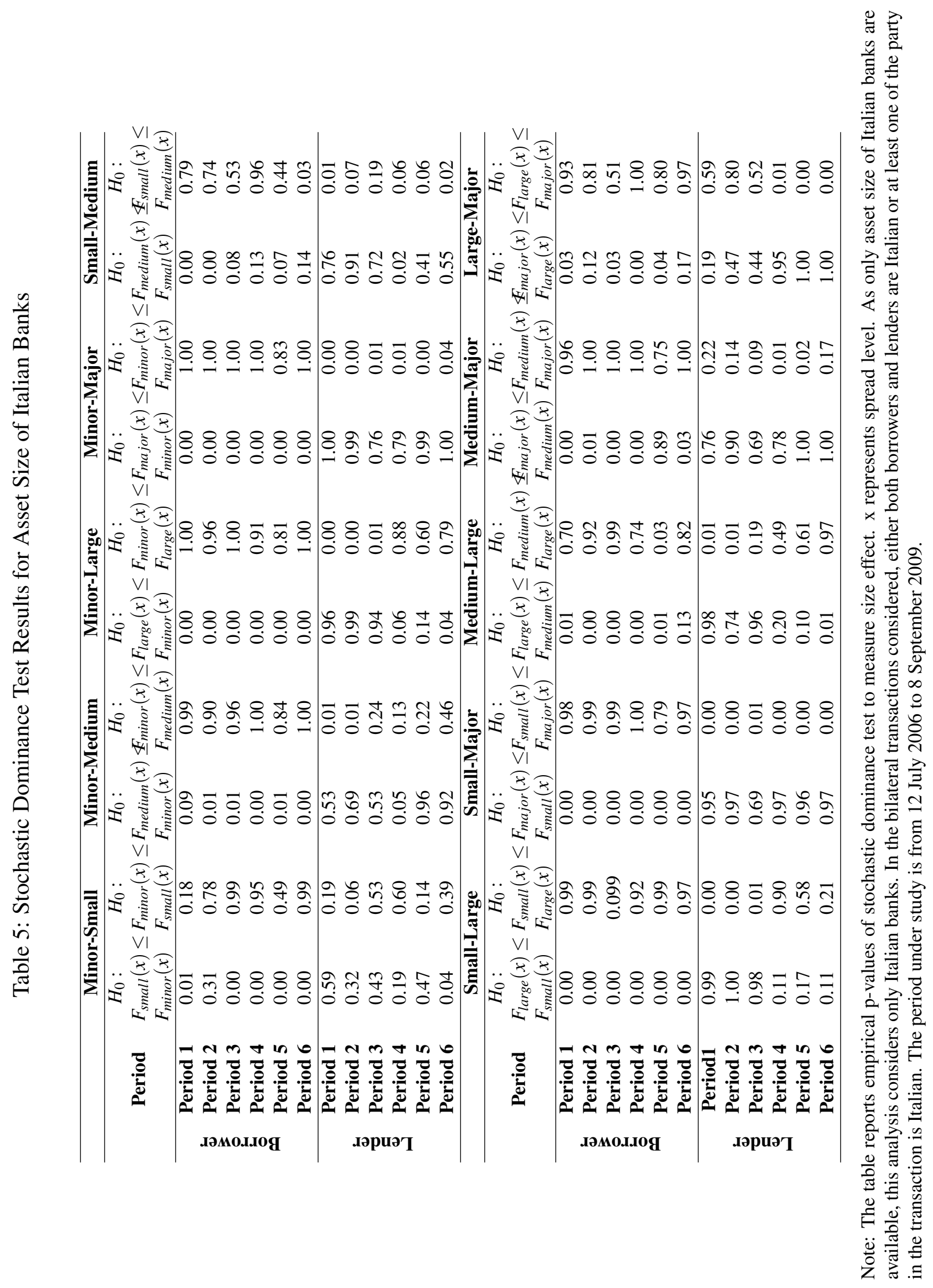




\subsection{Currency Effects}

The number of active countries in the e-MID market over our sample is sixteen. Most of these countries: Austria, Belgium, France, Germany, Greece, Ireland, Luxembourg, Netherlands, Portugal and Spain, have adhered the Euro currency. A few other participating countries such as United Kingdom, Denmark, Norway, Poland and Switzerland do not use the common currency. Commercial banks in the second group of countries are not allowed to open a Euro account with the ECB, and hence they cannot resort to the ECB deposit facilities. Figures 3 and 4 describe the densities of interbank spreads conditional on the main currency on which the bank operates. The graphs show no differences in funding rates until the second phase of the crisis (Lehman Brothers collapse). After it, the nonparametric densities indicate larger funding costs for banks in Non-Euro countries than for banks in countries using the Euro as main currency. The comparison of lending spreads between Euro and Non-Euro countries does not provide conclusive evidence over the six periods.

To provide further support to these findings obtained from the nonparametric estimation of the distribution and density functions, we perform first order stochastic dominance tests between these two groups of countries. The results presented in Panel A of Table 6 are consistent with the above discussion. The distribution function of borrowing rates of Euro countries is dominated by the distribution function of Non-Euro countries. This finding becomes statistically significant coinciding with the collapse of Lehman Brothers and thereafter up to period six. The results are reversed for the lending rates: the distribution of lending spreads of Euro countries dominates the counterpart distribution of Non-Euro countries during periods three and four, implying that the probability of setting large lending spreads is higher for Euro countries than Non-Euro countries over the crisis period. The lower lending rates offered by non Euro participants can be explained by the fact that these market participants do not have access to standing facilities of the ECB. Rather than converting their excess liquidity in their own currency, deposit it at their home central banks' overnight deposit facility, facing currency risk, it could be more profitable for these banks to lend their money abroad, at lower rates. These results are consistent with those reported by Heijmans, Heuver and Walraven (2010) and by Akram and Christophersen (2010) who infer data from the TARGET2 payment system respectively, for the Dutch and Norwegian interbank markets. These authors find that in some cases foreign banks' lending rates can even be below the central bank deposit rates.

\subsection{Sovereign Debt Effects}

The study of bank nationality on the cross-sectional distribution of spreads has recently gained importance given the unprecedented increase in spreads observed in some European sovereign debt markets. Countries exposed to sovereign crises are Greece, Ireland, Portugal and Spain. A comparison of money market developments between countries at the core of the euro area (Germany, France, The Netherlands, Belgium and Finland) and countries at the periphery, facing a sovereign debt crisis, has been reported by Arciero

et al. (2013). This study is based on interbank transactions inferred from TARGET2 data via the Furfine 
Table 6: Stochastic Dominance Test Results for Currency and Sovereign Debt Effects

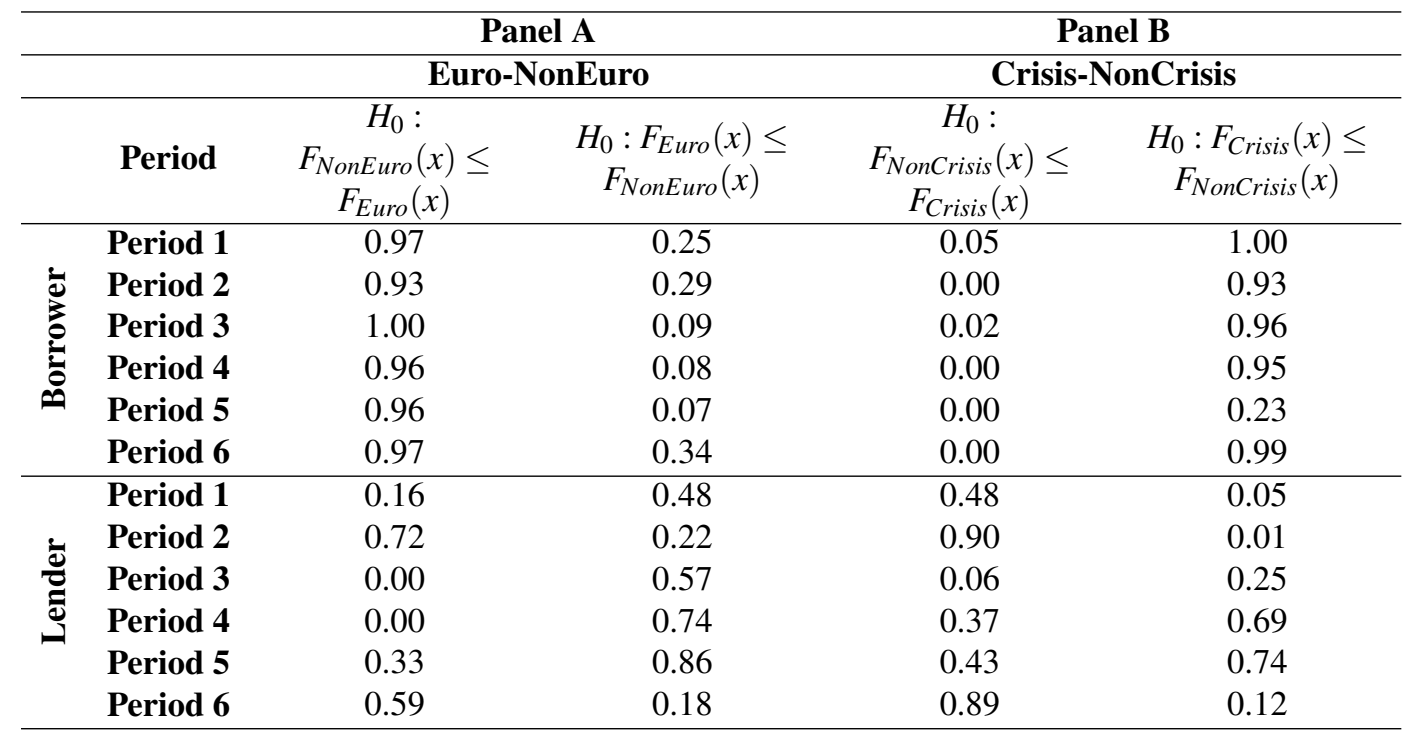

Note: The table reports empirical p-values of stochastic dominance test to measure currency and sovereign debt effect. $\mathrm{x}$ represents spread level. The period under study is from 12 July 2006 to 8 September 2009.

(1999) algorithm and cover the period from June 1st 2008 until October 31th 2012. The authors find that the periphery banks start paying higher rates than the core banks, after the Lehman default. Our analysis, as reported in Figures 5 and 6, shows differences in borrowing conditions already at the early stage of the financial crisis. After the collapse of Lehman Brothers banks in countries under sovereign distress experience borrowing rates well above those of banks in undistressed economies. The lending side, on the other hand, does not reflect significant differences in funding rates between banks in different economies. These findings provide empirical evidence of the existence of borrowing difficulties for banks in distressed countries well before their respective countries had trouble in funding themselves, and highlight the importance of the interbank market as an early warning indicator of sovereign debt distress.

This evidence is further robustified by testing for first order stochastic dominance between countries from the crisis group: Greece, Ireland, Portugal and Spain, and countries outside it such as Germany and U.K. The latter two countries are considered as the Non-Crisis group. Panel B of Table 6 reports overwhelming evidence of first order stochastic dominance of the random variable reflecting borrowing spreads of the Crisis group over the random variable corresponding to the Non-Crisis group. For the lending side, on the other hand, we do not observe statistical evidence to reject the equality of distributions between the spreads on the Crisis and Non-Crisis groups. 


\subsection{Nationality Effects}

The analysis of country-specific effects can be refined by conditioning on nationality and not only on a crisis/noncrisis classification. To do this we select Germany, Greece and U.K. as a representative example of the types discussed above. Germany represents banks from countries in the EU core economies, Greece represents banks from troubled economies in recent times and inside the EU, and U.K. describes a core economy that uses a different currency and hence it is outside the monetary system and ECB funding facilities. Table 7 presents interesting findings: borrowing spreads of British banks dominate stochastically borrowing spreads of German banks. This result is reversed for lending spreads, German banks enjoy larger lending spreads than their British counterparts. These two results combined suggest that U.K. banks are perceived as riskier than EU banks in core economies. For the case of British banks this may be due to their greater exposure to the American credit market than EU banks or, alternatively, to the choice of a different operating currency as pointed out in the previous analysis. To disentangle these effects it could be interesting to compare U.K. banks funding rates against other banks operating in EU countries outside the monetary system, however, for sake of space we do not perform such analysis and leave it as a possible extension.

Table 7: Stochastic Dominance Test Results for Nationality Effects

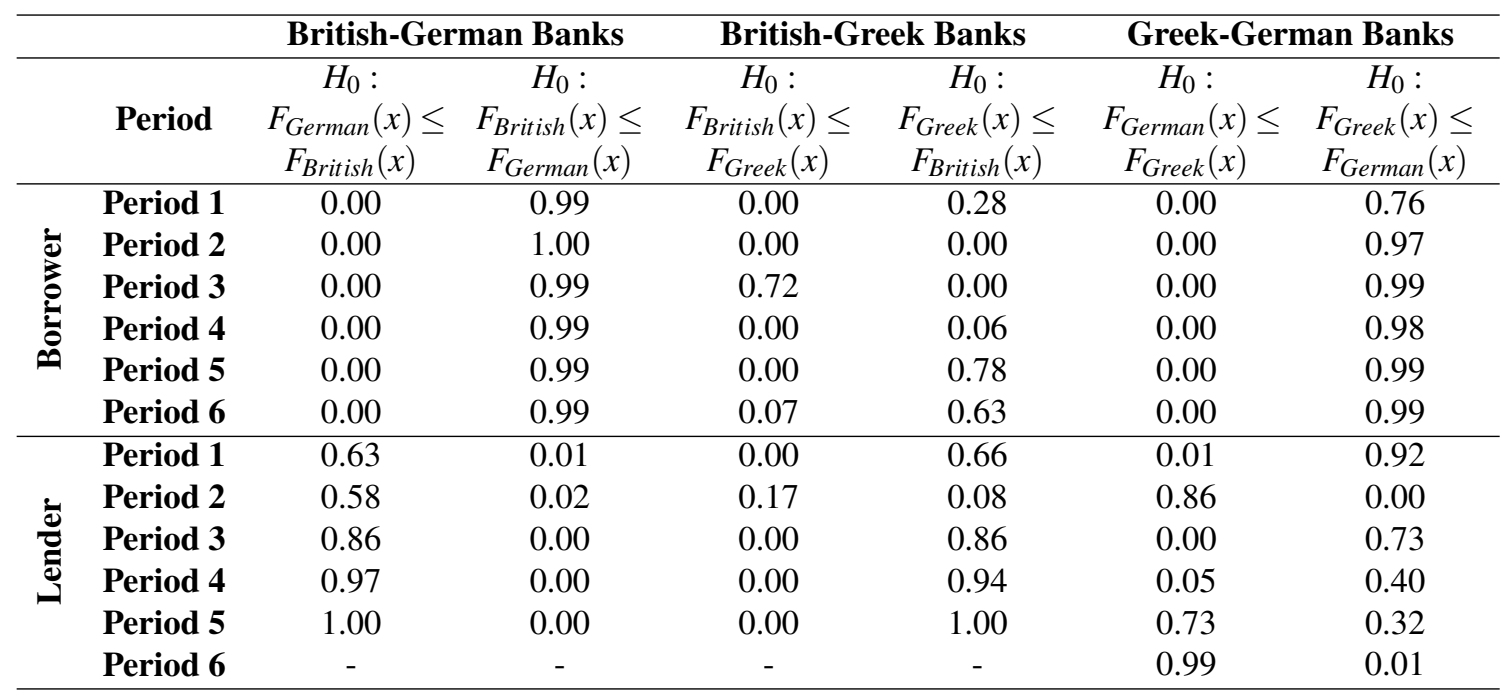

Note: The table reports empirical p-values of stochastic dominance test to measure nationality effect. $\mathrm{x}$ represents spread level. The period under study is from 12 July 2006 to 8 September 2009.

The case of Greek banks sheds similar findings. German banks are dominated stochastically by Greek banks for both borrowing and lending segments. The comparison against U.K. banks yields mixed results. The poor performance of Greek banks against British banks in terms of funding spreads is only statistically significant after September 2008. This period corresponds to the collapse of Lehman Brothers and the 
unfolding of the banking and sovereign crises that has affected Greece thereafter. Figures 7 and 8 illustrate the differences in the cross-section of borrowing conditions between banks based in Germany, Greece and United Kingdom.

\subsection{Calendar Effects}

According to Gaspar, Quiros and Mendizabal (2008), at least for the Eonia panel, all the cross section dispersion in spreads is realized on the last 2 days of the reserve maintenance period. To assess this with our dataset we have repeated our nonparametric exercise consisting of estimating the unconditional kernel density estimates for both the borrowing and lending markets. In Figures 9 and 10, we compare the densities of all transactions executed over the reserve maintenance period with a battery of new kernel density estimates computed from the transactions executed on the last two trading days of each maintenance period. More specifically, we plot the distributions of the average of the transactions of each borrower/lender bank over the last two days. As already shown in Gaspar, Quiros and Mendizabal (2008), the results suggest that the cross-sectional dispersion of funding rates is wider for the transactions executed over the last two trading days compared to the transactions executed over the whole reserve maintenance period. This empirical finding holds until the collapse of Lehman Brothers. After this event, the dispersion described by the nonparametric kernel density estimates for the whole maintenance period is similar to the dispersion corresponding to the last two days of trading. A potential explanation for these findings is given by the composition of the spreads. Before the collapse of Lehman Brothers, the spread can be interpreted as the combination of a credit risk effect and a liquidity risk effect. The liquidity effect explains the widening of the spread at the end of the maintenance period in periods where credit risk is small and its contribution to the spread negligible. After the Lehman event, the ECB injected plenty of liquidity so the liquidity effect most likely disappeared but the credit effect became more important. This may be the reason why the distribution of spreads for the whole maintenance period and the last two days of trading are similar after the Lehman event.

\subsection{Impact of Monetary Policy}

During the financial crisis, policy authorities worldwide implemented special measures to support liquidity and avoid the collapse of the financial system. In particular, the ECB enhanced credit support implementing five non-standard measures: the provision to Euro area banks of unlimited liquidity at a fixed rate in all refinancing operations against adequate collateral; the lengthening of the maximum maturity of refinancing operations from three months prior to the crisis to one year; the extension of the list of assets accepted as collateral; the provision of liquidity in foreign currencies (notably US dollars); the outright purchases in the covered bond market (European Central Bank, 2009).

From mid September 2007 to mid September 2008, the European Central Bank supplied greater amounts of liquidity at the beginning of the reserve maintenance period and reduced the liquidity surplus in the last 
days of such periods. The amounts offered in the longer-term refinancing operations registered an increase and, more generally, the ECB utilized a more flexible approach in the usual operations. In order to offer longer-term funds to the banking system, the Eurosystem in April and July 2008 arranged two longer-term refinancing operations having six months maturity. In September 2008 the ECB arranged four special fine tuning operations, injecting 165 billion Euros in the system; at the same time, it increased the volumes distributed through the main refinancing operations. On October 2008, the ECB decided to cut the official interest rate, carry out the weekly main refinancing operations through a fixed rate-full allotment procedures, to reduce the corridor of its standing facilities from 200 basis points to 100 basis points, and to expand the list of assets eligible for collateral, reducing to $B B B$ - the minimum rating for assets to be accepted. Between January and May 2009, the ECB Governing Council reduced the rate on the main refinancing operations by 150 basis points, to 1\%, in four steps. In May 2009 the ECB Governing Council agreed to conduct three liquidity providing longer-term refinancing operations (LTROs) with a maturity of 12 months in June, September and December 2009. In particular, the first 12-month LTRO, conducted on 24 June 2009, resulted in a record 442 billion Euros being allotted to the euro area banking system at a fixed rate of $1 \%$.

We test the effect of the two main interventions, the one in October 2008 and the one in June 2009, by estimating the cross-sectional kernel density estimates for the borrowing and lending segments with data corresponding to the months around October 2008 and June 2009. The results in Figures 11 to 14 uncover differences in the dispersion of spreads within each period and across the borrowing and lending segments. The reported graphs show that one implication of the monetary policy measures carried out in October 2008 and June 2009 is the reduction of the spreads in both tails of the distribution of funding rates. Note however that the reduction in the variability of spreads is more pronounced a few months after the implementation of such policies and not immediately after, capturing the stylized fact given by the delay of monetary policy in influencing the real economy.

\section{Conclusion}

Interbank markets are the main instrument for the transmission of monetary policy targets from central banks to the overall economy. This market is responsible for supplying liquidity to the financial system through the buying and selling operations of participant commercial banks. The cross-section of interbank rates provides useful information on the performance of the banking sector.

This article explores the cross-sectional distribution of monthly rates obtained as the average of daily spreads in the e-MID market over different periods covering the recent crises. We observe different dynamics in borrowing and lending rates before and after the occurrence of the crisis episodes in 2007-2008. Before the credit crunch crisis, we observe that the cross-section of spreads was narrow, revealing an efficient and well integrated market. This pattern radically changes after 2007 . The distribution of spreads becomes more disperse and multimodal in some cases. This stylized fact reflects the existence of clusters of banks facing similar funding conditions. Borrowing and lending rates exhibit similar patterns, the in- 
crease in dispersion is, however, more acute in the borrowing segment. After March 2009, and coinciding with a weak recovery of the financial sector, this tendency reverses and we observe the same patterns in the cross-section of interbank spreads as prior to the summer of 2007.

Our analysis also shows important differences in funding rates that can be tracked down to the extant variability in bank characteristics. Our exploratory analysis in the form of nonparametric density estimates of the cross-section of funding spreads and our statistical analysis based on first order stochastic dominance tests reveal interesting findings on the relationship between this variable and the operating currency, nationality, the economic region where banks operate and bank asset size. More specifically, banks in the periphery of the European Union face larger borrowing rates than EU core economies. Similarly, the adoption of the Euro acts as a shield against risk aversion for banks seeking funding. The empirical analysis also suggests that borrowing rates are a decreasing function of asset size and the group of largest banks benefit from being able to set higher than average lending rates.

Our analysis on calendar effects shows that the current institutional settings increase liquidity pressure on the last two days of the reserve maintenance period and in doing so put banks under distress, potentially increasing the fragility of the banking system on those dates. The fact that the calendar effect gradually disappears with the adoption of non-standard measures by the ECB is a clear indication that the widening of spread, mostly concentrated in these few days before the crisis, was mainly driven by poor liquidity management on the side of banks rather than by credit risk. The situation is reversed during the crisis, with liquidity pressure eased by the favourable funding conditions offered by the ECB, and spreads mostly reflecting different credit worthiness of participating banks. A policy implication of this analysis would be to encourage the design of a mechanism for reserve requirements that does not compel banks to simultaneously fulfill their average reserve.

As regards the decrease of the e-MID market turnover since the outbreak of the crisis, our results suggest that the lower volume and dropout of banks can be attributed to the crowding out of interbank intermediation, following the ECB exceptional intervention, rather than to a selection bias and flight to opacity, aimed to avoid the stigma effect possibly associated with the demand for liquidity in a transparent platform.

Our approach, by estimating the entire distribution of cross-sectional spreads, and not only mean and variance, provides a valuable tool for policy makers to monitor the soundness of the financial system. The widening of cross-sectional spread is an indicator of trust evaporation in the banking system. Thus monitoring the evolution of the spread distributions may help as an early warning indicator of a financial turmoil and to assess the success of policy intervention and quantitative easing programmes. 


\section{References}

[1] Acharya, V. V., D. Gromb and T. Yorulmazer. 2008. "Imperfect Competition in the Interbank Market for Liquidity as a Rationale for Central Banking." American Economic Journal: Macroeconomics 4(2) : $184-217$

[2] Acharya, V. V., and O. Merrouche. 2013. "Precautionary Hoarding of Liquidity and Inter-bank Markets: Evidence from the Sub-prime Crisis.” Review of Finance 17(1) :107-160.

[3] Acharya, V. V. and T. Yorulmazer. 2008. "Information Contagion and Bank Herding." Journal of Money, Credit and Banking 40 : 215-231.

[4] Afonso, G., A. Kovner and A. Schoar. 2011. "Stressed, Not Frozen: The Federal Funds Market in the Financial Crisis." Journal of Finance 66 : 1109-39.

[5] Aitchison,J. and C. G. G. Aitken. 1976. "Multivariate Binary Discrimination by the Kernel Method." Biometrika 63 : 413-420.

[6] Akram, F. A. and C. Christophersen. 2010. "Interbank Overnight Rates - Gains from Systemic Importance." Working Paper No. 11, Norges Bank.

[7] Allen, L. and A. Saunders. 1986. "The Large-Small Bank Dichotomy in the Federal Funds Market." Journal of Banking and Finance 10 : 219-230.

[8] Angelini, P., G. Mariesca and D. Russo. 1996. "Systemic Risk in the Netting System." Journal of Banking and Finance $20: 853-868$.

[9] Angelini, P. 2000. "Are Banks Risk-averse? A Note on the Intraday Timing of Operations in the Interbank Market." Journal of Money, Credit, and Banking 32 : 54-73.

[10] Angelini, P., A. Nobili and C. Picillo. 2011. "The Interbank Market after August 2007: What Has Changed and Why?" Journal of Money, Credit and Banking 43 : 923-958.

[11] Arciero, L. 2010. "Extracting Information on the Interbank Market from Payment System Data." Mimeo, Bank of Italy.

[12] Arciero, L., R. Heijmans, R. Heuver, M. Massarenti, C. Picillo and F. Vacirca. 2013. "How to Measure the Unsecured Money Market? Eurosystem's Implementation and Validation using TARGET2 Data." Working Paper No. 367, De Nederlandsche Bank.

[13] Ashcraft, A., J. McAndrews and D. Skeie. 2011. "Precautionary Reserves and the Interbank Market." Journal of Money, Credit and Banking 43 : 311-348. 
[14] Baglioni, A., A. Monticini. 2008. "The Intraday Price of Money: Evidence from the e-MID Interbank Market." Journal of Money, Credit and Banking 40 : 1533-1540.

[15] Baglioni, A. and A. Monticini. 2010. "The Intraday Interest Rate under a Liquidity Crisis: The Case of August 2007." Economics Letters 107(2) : 198-200.

[16] Baltagi, B.H. 2008. Econometric Analysis of Panel Data. West Sussex : Johhn Wiley \& Sons.

[17] Barrett, G. and S. Donald. 2003. "Consistent Tests for Stochastic Dominance." Econometrica 71 : 71-104.

[18] Beaupain, R., and A. Durre. 2011. 'Inferring Trading Dynamics for an OTC Market: The Case of the Euro Area Overnight Money Market." Quantitative Finance 11 (9) : 1285 - 1295.

[19] Beaupain, R. and A. Durr. 2013. "Central Bank Reserves and Interbank Market Liquidity in the Euro Area." Journal of Financial Intermediation 22 : 259-284.

[20] Bhattacharya, S. and D. Gale. 1987. "Preference Shocks, Liquidity and Central Bank Policy" In New Approaches to Monetary Economics, edited by W. Barnett, and K. Singleton, 69-88. New York: Cambridge University Press.

[21] Blavarg, M. and P. Nimander. 2002. "Interbank Exposures and Systemic Risk." Sveriges Riksbank Economic Review 2 : 19-45.

[22] Brousseau, V., A. Manzanares. 2005. "A Look at Intraday Frictions in the Euro Area Overnight Deposit Market.” Working Paper No. 439, European Central Bank.

[23] Brunetti, C., M. Filippo and J. H. Harris. 2011. "Effects of Central Bank Intervention on the Interbank Market During the Subprime Crisis." Review of Financial Studies 24(6) : 2053-2083.

[24] Buja, A., T. Hastie, and R. Tibshirani. 1989. "Linear Smoothers and Additive Models." Annals of Statistics 17 : 453-555.

[25] Cassola, N., Holthausen, C., and M. L. Duca. 2010. "The 2007/2009 Turmoil: A Challenge for the Integration of the Euro Area Money Market?" Mimeo. European Central Bank.

[26] Chernozhukov, V., S. Lee, and A. Rosen. 2013. "Intersection Bounds: Estimation and Inference." Econometrica 81(2) : 667737.

[27] Cocco J. F., F. J. Gomes and N. C. Martins. 2009. "Lending Relationships in the Interbank Market." Journal of Financial Intermediation 18 : 24-48.

[28] Davidson, R. and J. Y. Duclos. 2000. "Statistical Inference for Stochastic Dominance and for the Measurement of Poverty and Inequality." Econometrica 68 :1435 - 1464. 
[29] Diamond, D.W. and P.H. Dybvig. 1983. "Bank Runs, Deposit Insurance and Liquidity." Journal of Political Economy 91 : 401-419.

[30] Doukhan, P., P. Massart, and E. Rio. 1995. "Invariance Principles for Absolutely Regular Empirical Processes." Annales de l'Institut H. Poincare 31 : 393-427.

[31] Eisenschmidt, J. and J. Tapking. 2009. "Liquidity Risk Premia in Unsecured Interbank Money Markets.” Working Paper No. 1025, European Central Bank.

[32] European Central Bank , 2009. "Annual Report." Accessed October 15, 2014. http://www.ecb.europa.eu/pub/pdf/annrep/ar2009en.pdf.

[33] Finger, K., D. Fricke and T. Lux. 2012. "Network Analysis of the e-MID Overnight Money Market: The Informational Value of Different Aggregation Levels for Intrinsic Dynamic Processes." Working Paper No. 1782, Kiel Institute.

[34] Fishburn, P.C. 1977. "Mean-Risk Analysis with Risk Associated with Below-Target Returns." The American Economic Review 67 : 116-126.

[35] Freixas, X., B. M. Parigi and J. Rochet. 2000. "Systemic Risk, Interbank Relations and Liquidity Provision by the Central Bank." Journal of Money, Credit and Banking 32 : 611-638.

[36] Freixas, X. and C. Holthausen. 2004. "Interbank Market Integration under Asymmetric Information." Review of Financial Studies 18 (2) : 459-490.

[37] Freixas, X.B. and J. Jorge. 2008. "The Role of Interbank Markets in Monetary Policy: A Model with Rationing." Journal of Money, Credit and Banking 40 : 1151-1176.

[38] Furfine, C. H. 1999. 'The Microstructure of the Federal Funds Market." Financial Markets, Institutions and Instruments $8: 24-44$.

[39] Furfine, C. H. 2001. "Banks as Monitors of Other Banks: Evidence from the Overnight Federal Funds Market." The Journal of Business 74 : 33-57.

[40] Gabbi, G., G. Germano, B. Kapar, V. Hatzopoulos, G. Iori and M Politi. 2014. "Market Microstructure, Banks' Behaviour, and Interbank Spreads." Accessed October 15, 2014. http://openaccess.city.ac.uk/3950/

[41] Gabrieli, S. 2011a. "The Functioning of the European Interbank Market during the 2007-2008 Financial Crisis." Working Paper No. 158, CEIS Tor Vergata.

[42] Gabrieli, S. 2011b. "The Microstructure of the Money Market Before and After the Financial Crisis: A Network Perspective.” Working Paper No. 181, CEIS Tor Vergata. 
[43] Gaspar, V., G. P. Quiros and H. R. Mendizabal. 2008. "Interest Rate Dispersion and Volatility in the Market for Daily Funds." European Economic Review 52 : 413-440.

[44] Gonzalo, J. and J. Olmo. 2014. "Conditional Stochastic Dominance Tests." International Economic Review 55(3) : 819-838.

[45] Hall, P. Q. Li and J. Racine. 2004. "Cross-Validation and the Estimation of Conditional Probability Densities." Journal of the American Statistical Association 99 : 1015-1026.

[46] Hansen, B.E. 1996. 'Inference when a Nuisance Parameter is not Identified Under the Null Hypothesis." Econometrica 64 : 413-430.

[47] Hastie, T., and R. Tibshirani. 1990. Generalized Additive Models. London: Chapman and Hall.

[48] Heider, F., Hoerova, M., and C. Holthausen. 2009. "Liquidity Hoarding and Interbank Market Spreads: The Role of Counterparty Risk.” Working Paper No. 1126, European Central Bank.

[49] Heijmans, R., R. Heuver and D. Walraven. 2010. "Monitoring the Unsecured Interbank Money Market using TARGET2 Data.” Working Paper 276, De Nederlandsche Bank.

[50] Ho, T. and A. Saunders. 1985. "A Micro-Model of the Federal Funds Market." Journal of Finance 40 : 977-990.

[51] Iori, G., O. V. Precup. 2007. "Weighted Network Analysis of High-frequency Cross-correlation Measures."Physical Review 75 : 36-110.

[52] Iori, G., R. Ren, G. D. Masi, G. Caldarelli. 2007. "Trading Strategies in the Italian Interbank Market." Physica 376 : 467-479.

[53] Iori, G., G.D. Masi, O. V. Precup, G. Gabbi and G. Caldarelli. 2008. "A Network Analysis of the Italian Overnight Money Market." Journal of Economic Dynamics \& Control 32 : 259-278.

[54] Jun, S. J., Y. Lee and Y. Shin. 2011. ’Testing for Distributional Treatment Effects: A Set Identification Approach.” Working Paper.

[55] Li, Q. and J. S. Racine. 2008. "Nonparametric Estimation of Conditional CDF and Quantile Functions with Mixed Categorical and Continuous Data." Journal of Business and Economic Statistics 26 : 423 434.

[56] Linton, O., E. Maasoumi and Y.J. Whang. 2005. "Consistent Testing for Stochastic Dominance under General Sampling Schemes.” Review of Economic Studies 72 : 735-765. 
[57] Monticini, A. and F. Ravazzolo. 2014."Forecasting the Intraday Market Price of Money." Journal of Empirical Finance. Accessed September 6, 2014. ISSN 0927-5398. DOI: 10.1016/j.jempfin.2014.08.006.

[58] Nadaraya, E. A. 1965. "On Nonparametric Estimates of Density Functions and Regression Curves." Theory of Applied Probability $10: 186-190$.

[59] Newey, W.K. 1994. "Kernel Estimation of Partial Means and a General Variance Estimator." Econometric Theory $10: 233-253$.

[60] Northcott, C.A.s 2002. "Estimating Settlement Risk and the Potential for Contagion in Canada's Automated Clearing Settlement System.” Working Paper No: 41, Bank of Canada.

[61] Rochet, J.C. and J. Tirole. 1996. "Interbank Lending and Systemic Risk." Journal of Money, Credit and Banking $28: 733-762$.

[62] Scaillet, O., and N. Topaloglu. 2010. "Testing for Stochastic Dominance Efficiency." Journal of Business and Economic Statistics $28: 169-180$.

[63] Stigum, M., 1990. The Money Market. Third Edition. Homewood, Ill.: Dow Jones-Irwin.

[64] Upper, C. 2011. "Simulation Methods to Assess the Danger of Contagion in Interbank Markets." Journal of Financial Stability 7 : 111-125.

[65] van der Vaart, A.W., and J.A. Wellner. 1996. Weak Convergence and Empirical Processes. New York: Springer.

[66] Vento, G. A. and P. L. Ganga. 2010. "Interbank Market and Liquidity Distribution During the Great Financial Crisis : The e-MID Case." Journal of Money, Investment and Banking 18 : 68-94.

[67] Watson, G.S. 1964. "Smooth Regression Analysis." Sankhy: The Indian Journal of Statistics 26 (4) : 359-372. 


\section{Appendix: Proofs}

Proof of Theorem 1: For the sake of clarity in the presentation, we assume throughout the proof a balanced panel. There is no loss of generality in doing so.

(i) The proof of the consistency of (5) follows from the proof of Theorem 2.1 in Li and Racine (2008) for the cross-sectional case. More specifically, let $B_{j}(x, y)=\sum_{z \in Z} 1_{j}(z, x)\left[F_{z}(y) \mu(z)-F_{x}(y) \mu(x)\right] / \mu(x)$ with $Z$ the support of the covariate vector $I_{j, i t}$ and $1_{j}(z, x)=1(|z-x|=1) \prod_{j=1}^{k} 1(|z-x|=0)$; let $|h|=\sum_{j=1}^{k} h_{s}$. Our assumptions A.1-A.6 contain conditions (C1)-(C3) in Li and Racine (2008). These authors show that under these conditions

$$
\widehat{F}_{x}(y)=F_{x}(y)+\sum_{j=1}^{k} h_{j} B_{j}(x, y)+o\left(|h|^{2}\right),
$$

for any fixed pair $(x, y) \in \widetilde{\Omega}$. Now, under assumption A.6 the leading bias term converges to zero as $N \rightarrow \infty$ and the asymptotic consistency of the estimator follows.

(ii) To derive the asymptotic distribution of the standardized estimator we note that $E\left[n^{1 / 2}\left(\widehat{F}_{x}(y)-F_{x}(y)\right)\right]$ converges to zero in probability. This is so by assumption A.6 that imposes that $N^{1 / 2} h \rightarrow 0$ as $N \rightarrow \infty$ with $T$ fixed. Under this assumption the bias of the nonparametric estimator given by $\sum_{j=1}^{k} h_{j} B_{j}(x, y)$ converges to zero as $N$ increases.

To complete the proof we need to find the limiting variance of $n^{1 / 2}\left(\widehat{F}_{x}(y)-F_{x}(y)\right)$ as $N \rightarrow \infty$, and apply a Liapunov Central Limit Theorem. First, note that $n^{1 / 2}\left(\widehat{F}_{x}(y)-F_{x}(y)\right)=n^{1 / 2}\left(\widehat{F}_{x}(y)-F_{x}(y)\right) \widehat{\mu}(x) / \mu(x)+$ $o_{P}(1)$. Thus, $V\left(n^{1 / 2}\left(\widehat{F}_{x}(y)-F_{x}(y)\right)\right)=V\left(n^{1 / 2}\left(\widehat{F}_{x}(y)-F_{x}(y)\right) \widehat{\mu}(x)\right) / \mu^{2}(x)+o_{P}(1)$. Then

$$
\begin{gathered}
V\left(n^{1 / 2}\left(\widehat{F}_{x}(y)-F_{x}(y)\right) \widehat{\mu}(x)\right)=n^{-1} \sum_{i=1}^{N} \sum_{t=1}^{T} V\left(\left(d_{i t}(y)-F_{x}(y)\right) W_{h}\left(I_{i t} ; x\right)\right)+ \\
\left.n^{-1} \sum_{\substack { i=1 \\
\begin{subarray}{c}{s, t=1 \\
t \neq s{ i = 1 \\
\begin{subarray} { c } { s , t = 1 \\
t \neq s } }\end{subarray}}^{T} \operatorname{Cov}\left(d_{i s}(y)-F_{x}(y)\right) W_{h}\left(I_{i s} ; x\right),\left(d_{i t}(y)-F_{x}(y)\right) W_{h}\left(I_{i t} ; x\right)\right) .
\end{gathered}
$$

The first term in (13) is such that

$$
\begin{gathered}
V\left(\left(d_{i t}(y)-F_{x}(y)\right) W_{h}\left(I_{i t} ; x\right)\right)=E\left[\left(d_{i t}(y)-F_{x}(y)\right)^{2} W_{h}^{2}\left(I_{i t} ; x\right)\right]+O(|h|) \\
\left.=E\left[\left(\eta_{i}^{2}+F_{z}(y)-2 F_{z}(y) F_{x}(y)+F_{x}^{2}(y)\right) W_{h}^{2}\left(I_{i t} ; x\right)\right]+O(|h|)=\left(\sigma_{i}^{2}+F_{x}(y)\left(1-F_{x}(y)\right)\right) \mu(x)+O(|h|) .\right]
\end{gathered}
$$

The second term in (13) is

$$
\operatorname{Cov}\left(\left(d_{i s}(y)-F_{x}(y)\right) W_{h}\left(I_{i s} ; x\right),\left(d_{i t}(y)-F_{x}(y)\right) W_{h}\left(I_{i t} ; x\right)\right)=E\left[V\left(\mu_{i}\right) W_{h}\left(I_{i s} ; x\right) W_{h}\left(I_{i t} ; x\right)\right]+O(|h|)
$$




$$
=\sigma_{i}^{2} P\left\{I_{i s}=x, I_{i t}=x\right\}+O(|h|) .
$$

Then, assuming that $P\left\{I_{i s}=x, I_{i t}=x\right\}$ is the same across individuals and $n=N T$, expression (13) takes the following form:

$$
V\left(n^{1 / 2}\left(\widehat{F}_{x}(y)-F_{x}(y)\right) \widehat{\mu}(x)\right)=F_{x}(y)\left(1-F_{x}(y)\right) \mu(x)+N^{-1} \sum_{i=1}^{N} \sigma_{i}^{2}(\mu(x)+\lambda(x))+O(|h|),
$$

with $\lambda(x)=T^{-1} \sum_{\substack{s, t=1 \\ t \neq s}}^{T} P\left\{I_{i s}=x, I_{i t}=x\right\}$. Applying the law of large numbers to the iid cross section we obtain that

$$
\lim _{N \rightarrow \infty} V\left(n^{1 / 2}\left(\widehat{F}_{x}(y)-F_{x}(y)\right) \widehat{\mu}(x)\right)=F_{x}(y)\left(1-F_{x}(y)\right) \mu(x)+E\left[\sigma_{i}^{2}\right](\mu(x)+\lambda(x))+O(|h|),
$$

Using a Liapunov Central Limit Theorem, it follows that

$$
n^{1 / 2}\left(\widehat{F}_{x}(y)-F_{x}(y)\right) \stackrel{d}{\rightarrow} N\left(0, \Sigma_{x}(y)\right)
$$

with $\Sigma_{x}(y)=\left(F_{x}(y)\left(1-F_{x}(y)\right) / \mu(x)+E\left[\sigma_{i}^{2}\right](\mu(x)+\lambda(x)) / \mu^{2}(x)\right.$.

\section{Proof of Corollary 1:}

(i) The presence of a persistent estimator is defined in this case as $P\left\{I_{i t}=x \mid I_{i s}=x\right\}=1$ with $s<t$. The asymptotic variance $\Sigma_{x}(y)$ in Theorem 1 becomes

$$
\Sigma_{x}(y)=\left(F_{x}(y)\left(1-F_{x}(y)\right)+T \sigma^{2}\right) / \mu(x) .
$$

(ii) If the covariates are serially independent the asymptotic variance is

$$
\Sigma_{x}(y)=\left(F_{x}(y)\left(1-F_{x}(y)\right)+\sigma^{2}\right) / \mu(x)+(T-1) \sigma^{2} .
$$

Proof of Theorem 2: The function $S_{n}(y ; x, \widetilde{x})=n^{1 / 2}\left(\left(\widehat{F}_{x}(y)-F_{x}(y)\right)-\left(\widehat{F}_{\widetilde{x}}(y)-F_{\widetilde{x}}(y)\right)\right)$ can be expressed as $S_{n}(y ; x, \widetilde{x})=n^{-1 / 2} \sum_{i=1}^{N} \sum_{t=1}^{T} s_{i t}(y ; x, \widetilde{x})$ with

$$
s_{i t}(y ; x, \widetilde{x})=\left(d_{i t}(y)-F_{x}(y)\right) W_{h}\left(I_{i t} ; x\right) / \widehat{\mu}(x)-\left(d_{i t}(y)-F_{\widetilde{x}}(y)\right) W_{h}\left(I_{i t} ; \widetilde{x}\right) / \widehat{\mu}(\widetilde{x}) .
$$


Its empirical covariance function is $K_{n}\left(y_{1}, y_{2} ; x, \widetilde{x}\right)$ that is defined as

$$
K_{n}\left(y_{1}, y_{2} ; x, \widetilde{x}\right)=n^{-1} \sum_{i, j=1}^{N} \sum_{s, t=1}^{T} s_{i s}\left(y_{1} ; x, \widetilde{x}\right) s_{j t}\left(y_{2} ; x, \widetilde{x}\right)
$$

Under A.1-A.8, for each $y \in \Omega$ and fixed pair $(x, \widetilde{x}) \in \Omega^{*}, s_{i t}(y ; x, \widetilde{x})$ is a square integrable stationary sequence with an asymptotic mean equal to zero as $N \rightarrow \infty$, to which the pointwise central limit theorem applies. Furthermore, note that $s_{i t}(y ; x, \widetilde{x})=\widetilde{s_{i t}}(y ; x, \widetilde{x})+o_{P}(1)$, with

$$
\widetilde{s}_{i t}(y ; x, \widetilde{x})=\left(d_{i t}(y)-F_{x}(y)\right) W_{h}\left(I_{i t} ; x\right) / \mu(x)-\left(d_{i t}(y)-F_{\widetilde{x}}(y)\right) W_{h}\left(I_{i t} ; \widetilde{x}\right) / \mu(\widetilde{x}),
$$

and the covariance of $S_{n}(y ; x, \widetilde{x})$, defined as $E\left[S_{n}\left(y_{1} ; x, \widetilde{x}\right) S_{n}\left(y_{2} ; x, \widetilde{x}\right)\right]$, satisfies that

$$
E\left[S_{n}\left(y_{1} ; x, \widetilde{x}\right) S_{n}\left(y_{2} ; x, \widetilde{x}\right)\right]=E\left[\widetilde{S}_{n}\left(y_{1} ; x, \widetilde{x}\right) \widetilde{S}_{n}\left(y_{2} ; x, \widetilde{x}\right)\right]+o_{P}(1)
$$

with $\widetilde{S}_{n}(y ; x, \widetilde{x})=n^{-1 / 2} \sum_{i=1}^{N} \sum_{t=1}^{T} \widetilde{s}_{i t}(y ; x, \widetilde{x})$. The cross-sectional independence imposed on A.2 implies that the covariance kernel for $S_{n}(y \mid x, \widetilde{x})$ can be approximated by

$$
E\left[\widetilde{S}_{n}\left(y_{1} ; x, \widetilde{x}\right) \widetilde{S}_{n}\left(y_{2} ; x, \widetilde{x}\right)\right]=n^{-1} \sum_{i=1}^{N} \sum_{t=1}^{T} E\left[\widetilde{s}_{i t}\left(y_{1} ; x, \widetilde{x}\right) \widetilde{s}_{i t}\left(y_{2} ; x, \widetilde{x}\right)\right]+n^{-1} \sum_{\substack { i=1 \\
\begin{subarray}{c}{s, t=1 \\
t \neq s{ i = 1 \\
\begin{subarray} { c } { s , t = 1 \\
t \neq s } }\end{subarray}}^{T} E\left[\widetilde{s}_{i s}\left(y_{1} ; x, \widetilde{x}\right) \widetilde{s}_{i t}\left(y_{2} ; x, \widetilde{x}\right)\right]
$$

where

$$
\begin{aligned}
E\left[\widetilde{s}_{i t}\left(y_{1} ; x, \widetilde{x}\right) \widetilde{s}_{i t}\left(y_{2} ; x, \widetilde{x}\right)\right] & =\left(\sigma_{i}^{2}+F_{x}\left(\min \left(y_{1}, y_{2}\right)\right)-F_{x}\left(y_{1}\right) F_{x}\left(y_{2}\right)\right) / \mu^{2}(x) \\
& +\left(\sigma_{i}^{2}+F_{\widetilde{x}}\left(\min \left(y_{1}, y_{2}\right)\right)-F_{\widetilde{x}}\left(y_{1}\right) F_{\widetilde{x}}\left(y_{2}\right)\right) / \mu^{2}(\widetilde{x})+O(|h|),
\end{aligned}
$$

and

$$
E\left[\widetilde{s}_{i s}\left(y_{1} ; x, \widetilde{x}\right) \widetilde{s}_{i t}\left(y_{2} ; x, \widetilde{x}\right)\right]=\sigma_{i}^{2} P\left\{I_{i s}=x, I_{i t}=\widetilde{x}\right\} /(\mu(x) \mu(\widetilde{x}))+O(|h|) .
$$

Then,

$$
\begin{aligned}
E\left[\widetilde{S}_{n}\left(y_{1} ; x, \widetilde{x}\right) \widetilde{S}_{n}\left(y_{2} ; x, \widetilde{x}\right)\right] & =\left(F_{x}\left(\min \left(y_{1}, y_{2}\right)\right)-F_{x}\left(y_{1}\right) F_{x}\left(y_{2}\right)\right) / \mu^{2}(x) \\
& +\left(F_{\widetilde{x}}\left(\min \left(y_{1}, y_{2}\right)\right)-F_{\widetilde{x}}\left(y_{1}\right) F_{\widetilde{x}}\left(y_{2}\right)\right) / \mu^{2}(\widetilde{x}) \\
& +N^{-1} \sum_{i=1}^{N} \sigma_{i}^{2}\left(1 / \mu^{2}(x)+1 / \mu^{2}(\widetilde{x})\right) \\
& +N^{-1} \sum_{i=1}^{N} \sigma_{i}^{2} \lambda(x, \widetilde{x}) /(\mu(x) \mu(\widetilde{x}))+O(|h|),
\end{aligned}
$$


with $\lambda(x, \widetilde{x})=T^{-1} \sum_{\substack{s, t=1 \\ t \neq s}}^{T} P\left\{I_{i s}=x, I_{i t}=\widetilde{x}\right\}$.

The asymptotic covariance kernel of the limiting Gaussian process $S_{\infty}(y ; x, \widetilde{x})$ is

$$
\begin{aligned}
K_{\infty}\left(y_{1}, y_{2} ; x, \widetilde{x}\right) & =\left(F_{x}\left(\min \left(y_{1}, y_{2}\right)\right)-F_{x}\left(y_{1}\right) F_{x}\left(y_{2}\right)\right) / \mu^{2}(x)+\left(F_{\widetilde{x}}\left(\min \left(y_{1}, y_{2}\right)\right)-F_{\widetilde{x}}\left(y_{1}\right) F_{\widetilde{x}}\left(y_{2}\right)\right) / \mu^{2}(\widetilde{x}) \\
& +E_{c}\left[\sigma_{i}^{2}\right]\left(\mu^{2}(x)+\mu^{2}(\widetilde{x})+\lambda(x, \widetilde{x}) \mu(x) \mu(\widetilde{x})\right) /\left(\mu^{2}(x) \mu^{2}(\widetilde{x})\right) .
\end{aligned}
$$

The multivariate central limit theorem establishes the finite dimensional distributional convergence. To establish stochastic equicontinuity, we appeal to Theorem 1 of Doukhan, Massart and Rio (1995). By assumption A.2, the summands $s_{i t}(y ; x, \widetilde{x})$ satisfy the necessary absolute regularity mixing decay rate. Further, the envelope function $\sup _{y \in \Omega}\left|s_{i t}(y ; x, \tilde{x})\right|$ is bounded by construction of $d_{i t}(y)$ and the kernel functions $w_{h}\left(I_{j, i t} ; x_{j}\right)$ in $W_{h}\left(I_{i t} ; x\right)$. The rest of the proof follows from the proof of Theorem 1 in Hansen (1996).

Proof of Corollary 2: The proof is analogous to the proof for the cross-sectional case presented in Barrett and Donald (2003). Under A.1-A.8, the application of Theorem 2 and the continuous mapping theorem imply that

$$
\sup _{y \in \Omega}\left|S_{n}(y ; x, \widetilde{x})\right| \stackrel{d}{\rightarrow} \sup _{y \in \Omega}\left|S_{\infty}(y ; x, \widetilde{x})\right| .
$$

Let $c_{\alpha}$ denote the $(1-\alpha)$ quantile of the distribution of $\sup _{y \in \Omega}\left|S_{\infty}(y ; x, \widetilde{x})\right|$. This implies that

$$
\lim _{N \rightarrow \infty} P\left\{\sup _{y \in \Omega}\left|S_{n}(y ; x, \widetilde{x})\right|>c_{\alpha}\right\}=\alpha
$$

Further, it is not difficult to see that under the null hypothesis $H_{0}$, the statistic $T_{n}(x, \widetilde{x})$ is majorized by $\sup _{y \in \Omega}\left|S_{n}(y ; x, \widetilde{x})\right|$, hence,

$$
\lim _{N \rightarrow \infty} P\left\{T_{n}(x, \widetilde{x})>c_{\alpha}\right\} \leq \lim _{N \rightarrow \infty} P\left\{\sup _{y \in \Omega}\left|S_{n}(y ; x, \widetilde{x})\right|>c_{\alpha}\right\}=\alpha
$$

Under the least favourable case, it follows that $T_{n}(x, \widetilde{x})$ and $\sup _{y \in \Omega}\left|S_{n}(y ; x, \widetilde{x})\right|$ are the same process, hence the above condition holds with equality for all $y \in \Omega$.

Finally, to derive the consistency of the test under $H_{A}$, note that $T_{n}(x, \widetilde{x}) \rightarrow \infty$ as $N \rightarrow \infty$, implying that

$$
\lim _{N \rightarrow \infty} P\left\{T_{n}(x, \widetilde{x})>c_{\alpha}\right\}=1
$$



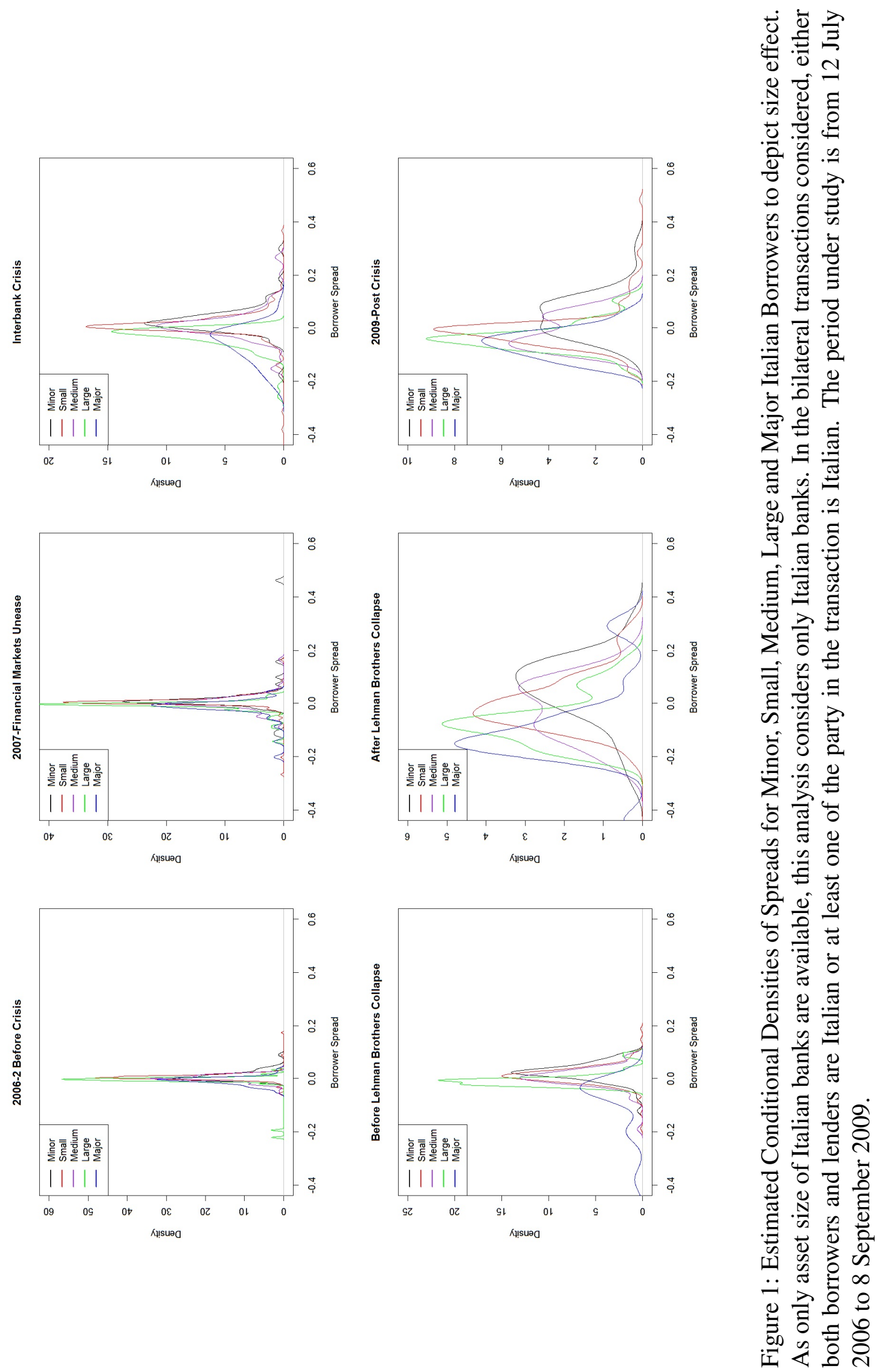

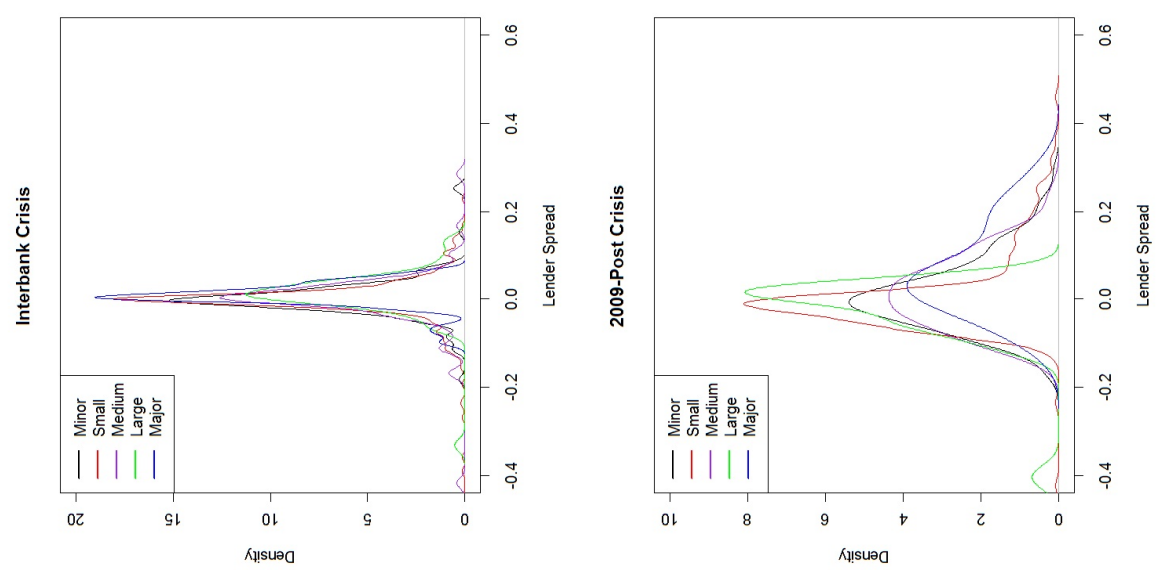

过㐫

ब $\mathrm{\sigma}$

岕 总

.

웡 응

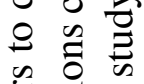

क⿺辶万

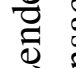

픙

.

.
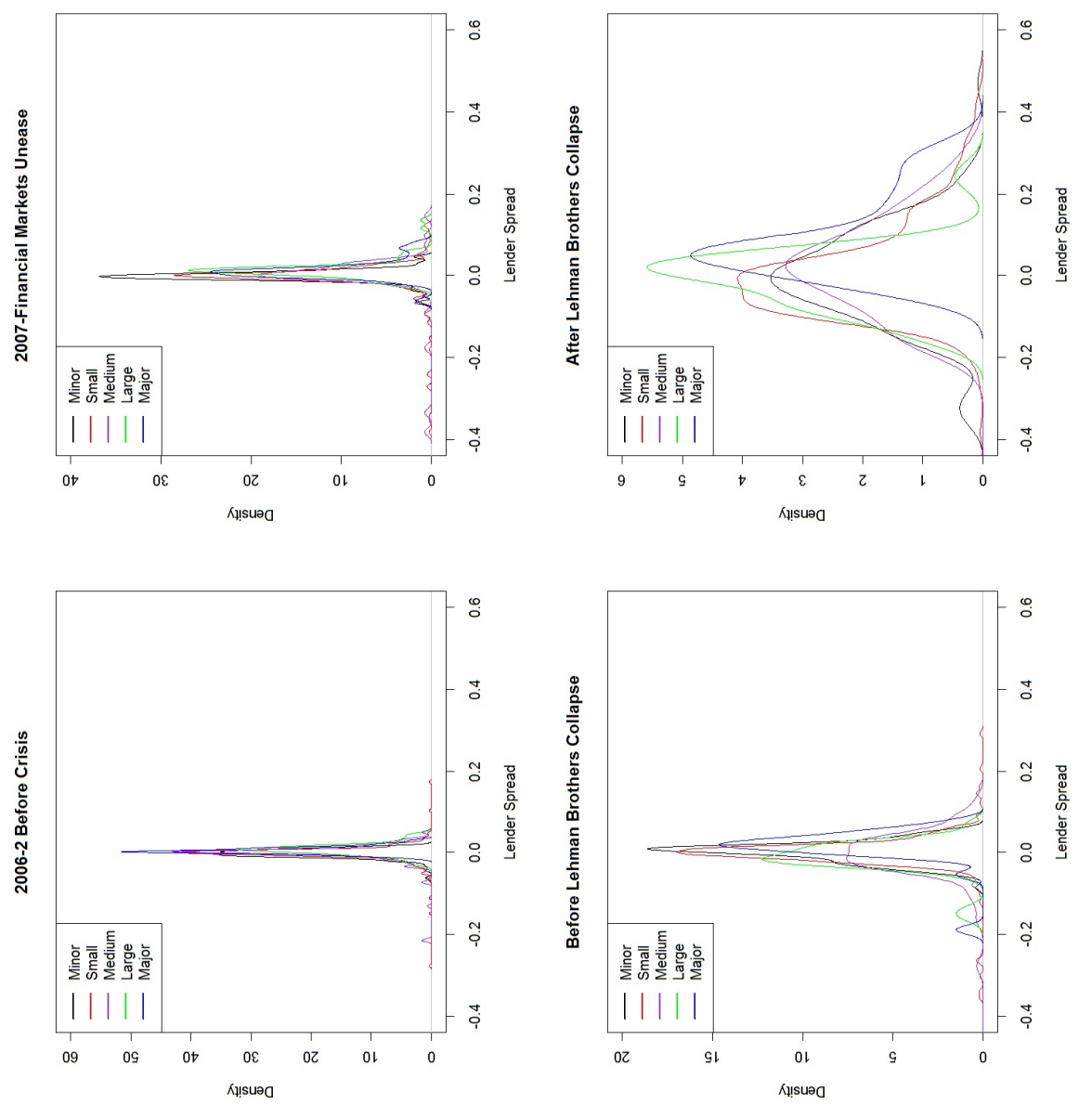

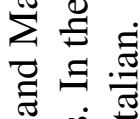

0 步

芚

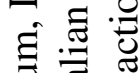

焉焉

$\Rightarrow$ 웜

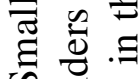

ज

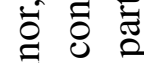

$\sum_{i=1}^{\infty}$

๖ำ눈

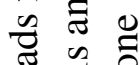

营过

फ

。

氙

졸

ีㅡㅇ

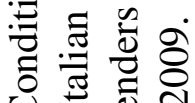

U

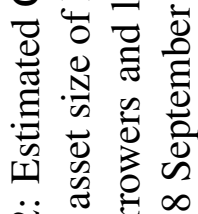

ن

용요

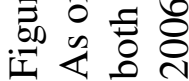



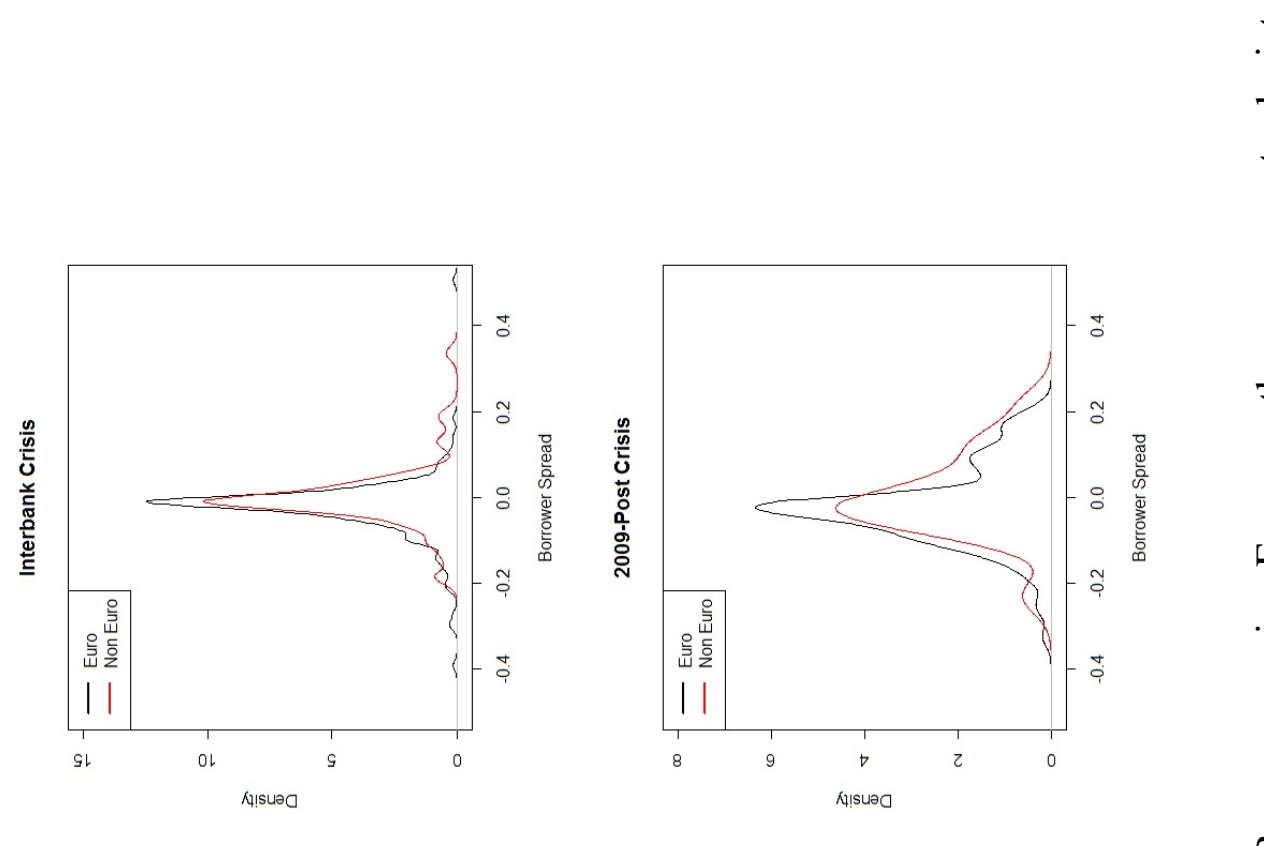

0
0
0
0
0
0
0
0
0
0
0
0
0
$\vdots$
0
0
0
0
0
0
0
0
0
0
0
0
0
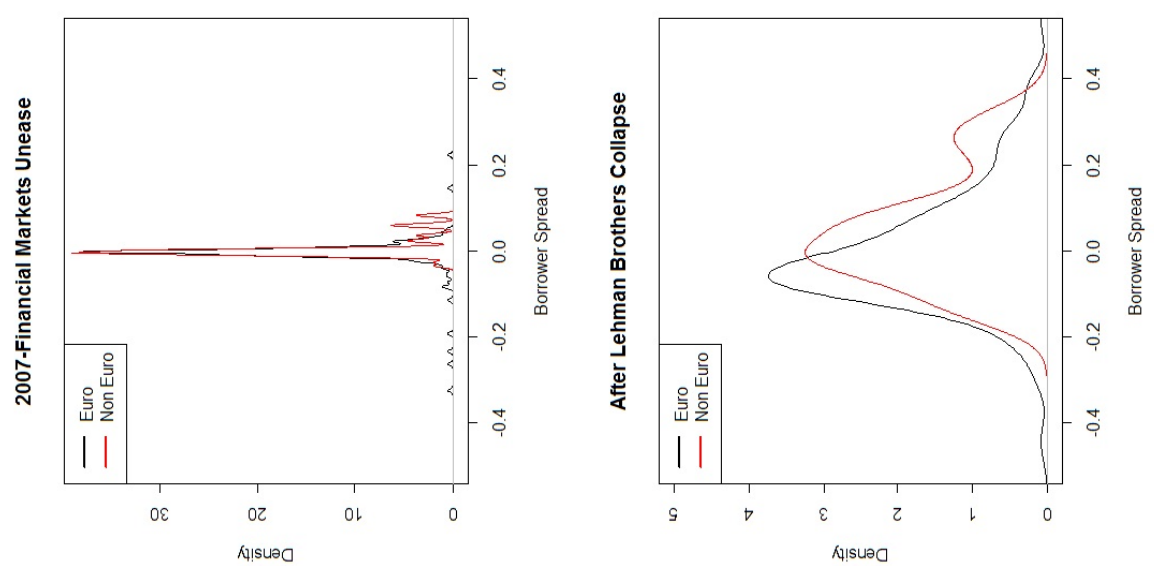

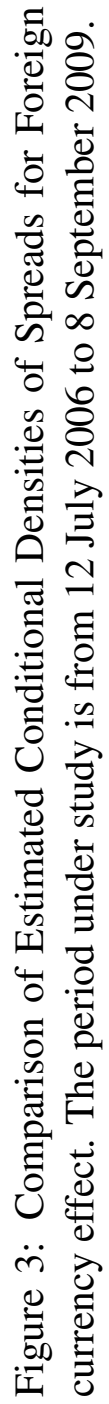
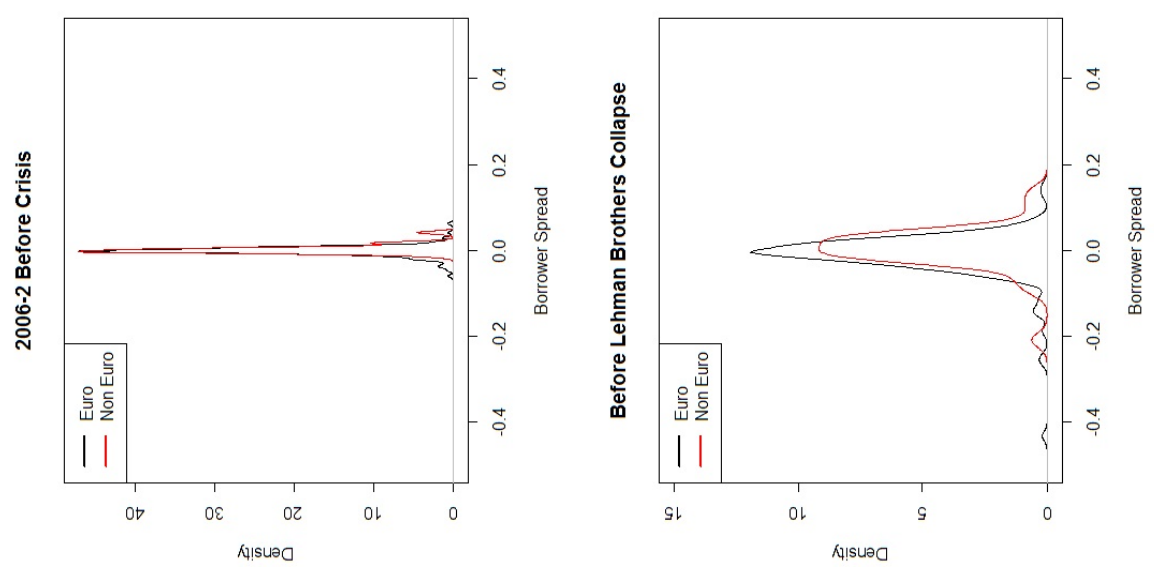

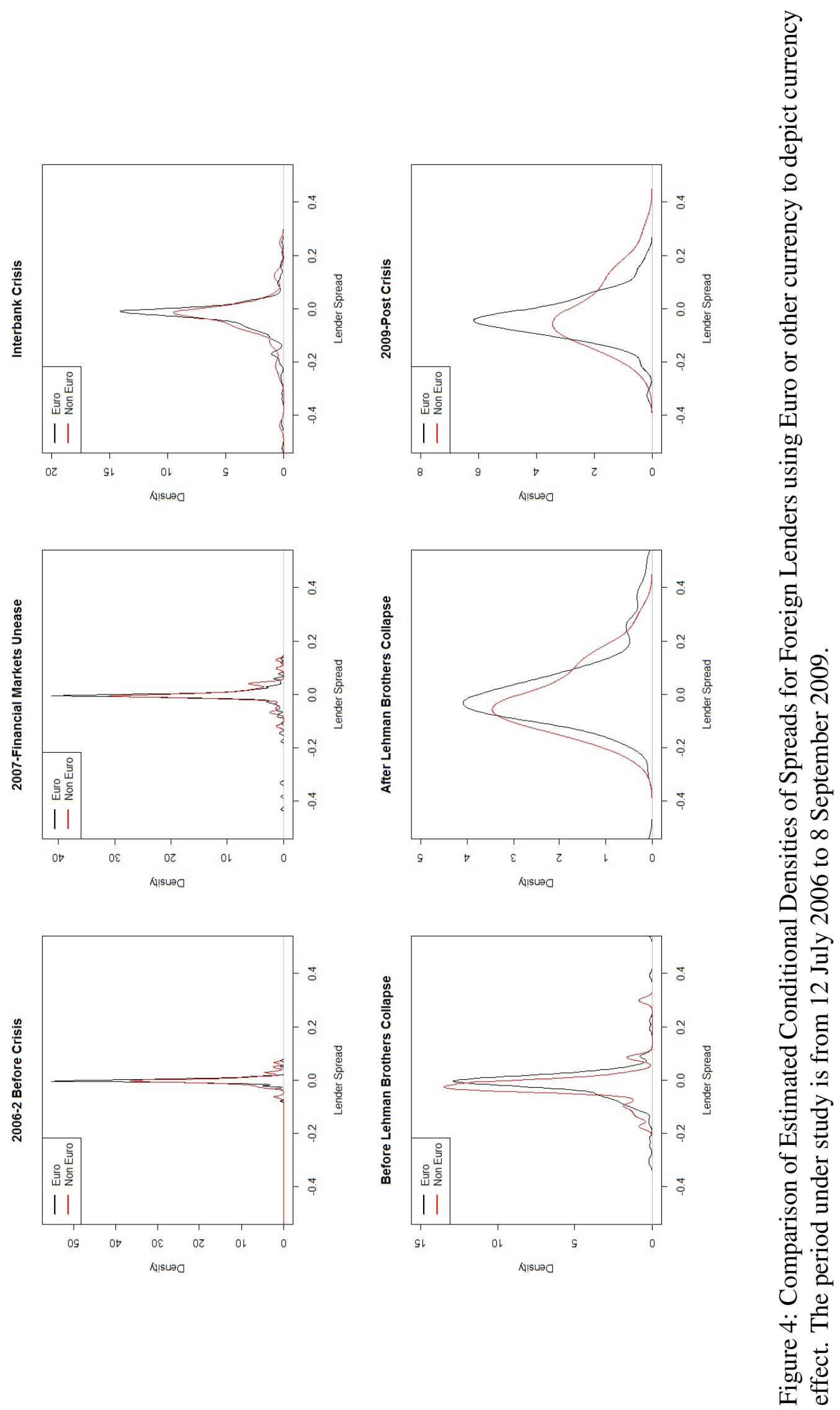

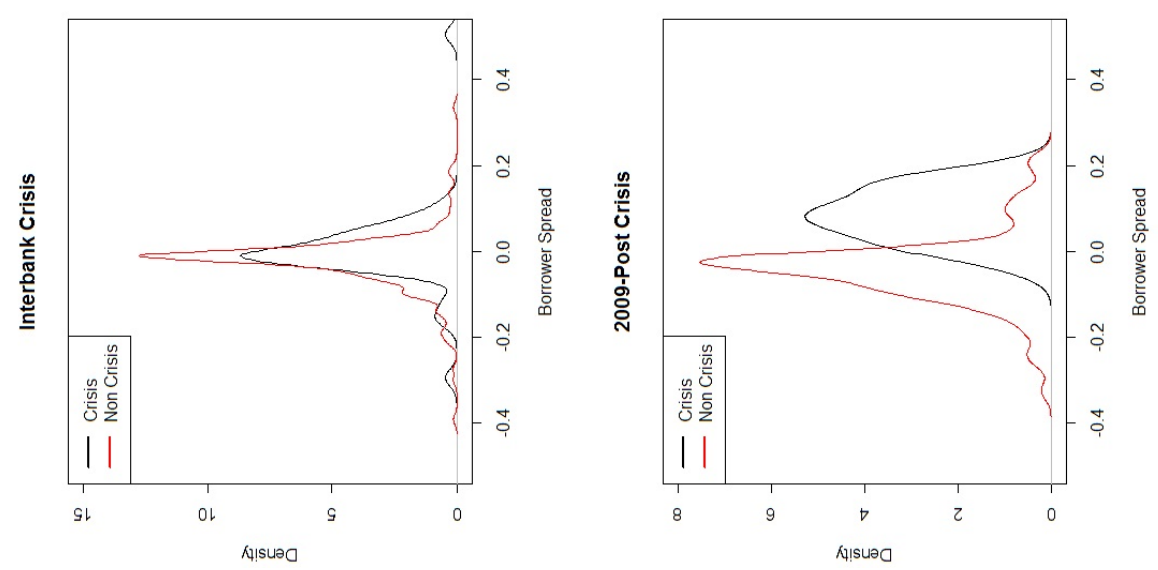

$\stackrel{0}{0}$
0
0
0
0
0
0
0
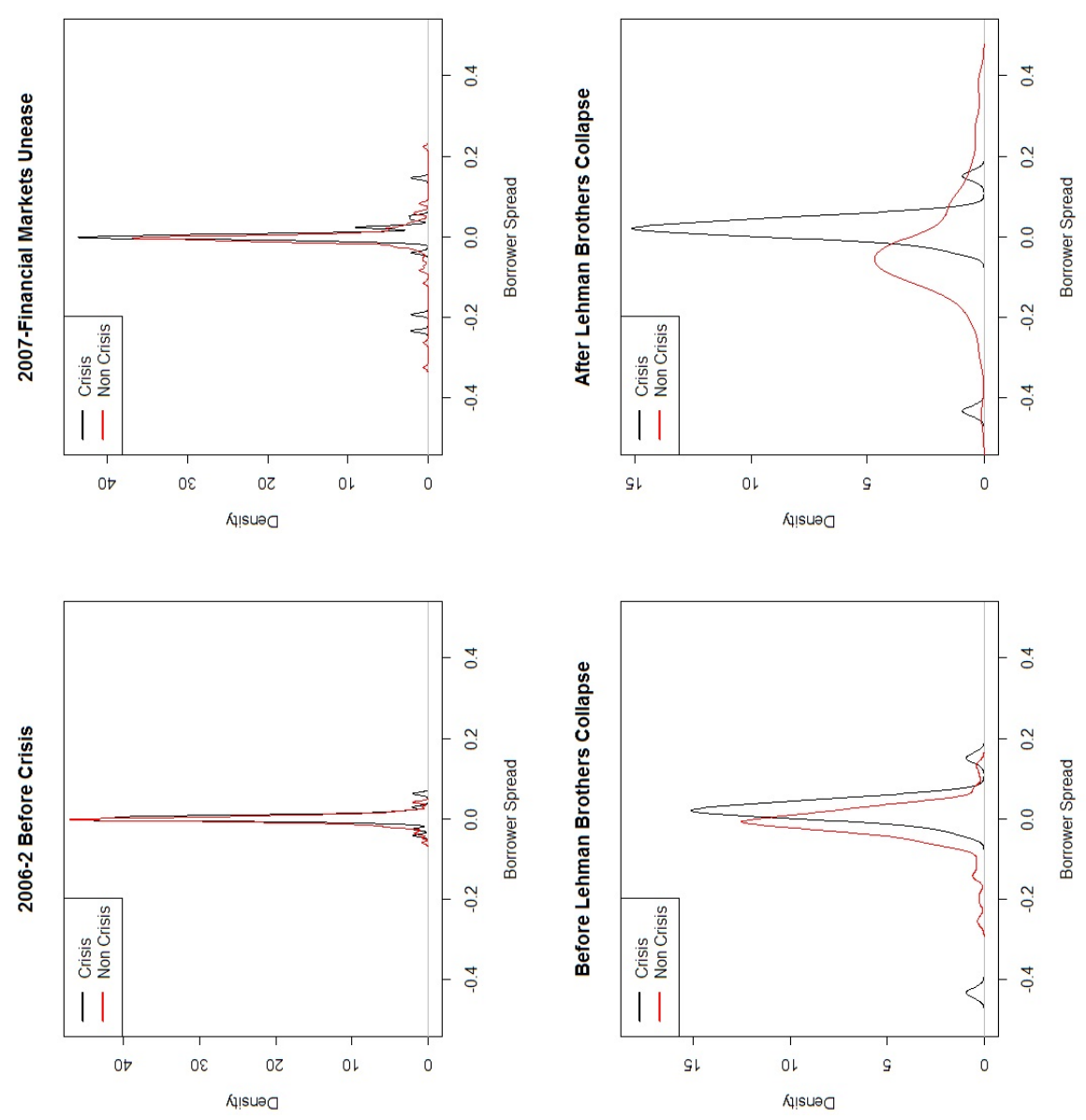

ह5

:

ฝै

ํㅡㅁ

है

.50

एँ

훈

용

흔

जै

:

ญ

드를

:气

पु

忥

II

प.

苛

유 해

ن

$\ddot{\text { iे }}$

$\stackrel{\Xi}{\Xi}$

i 

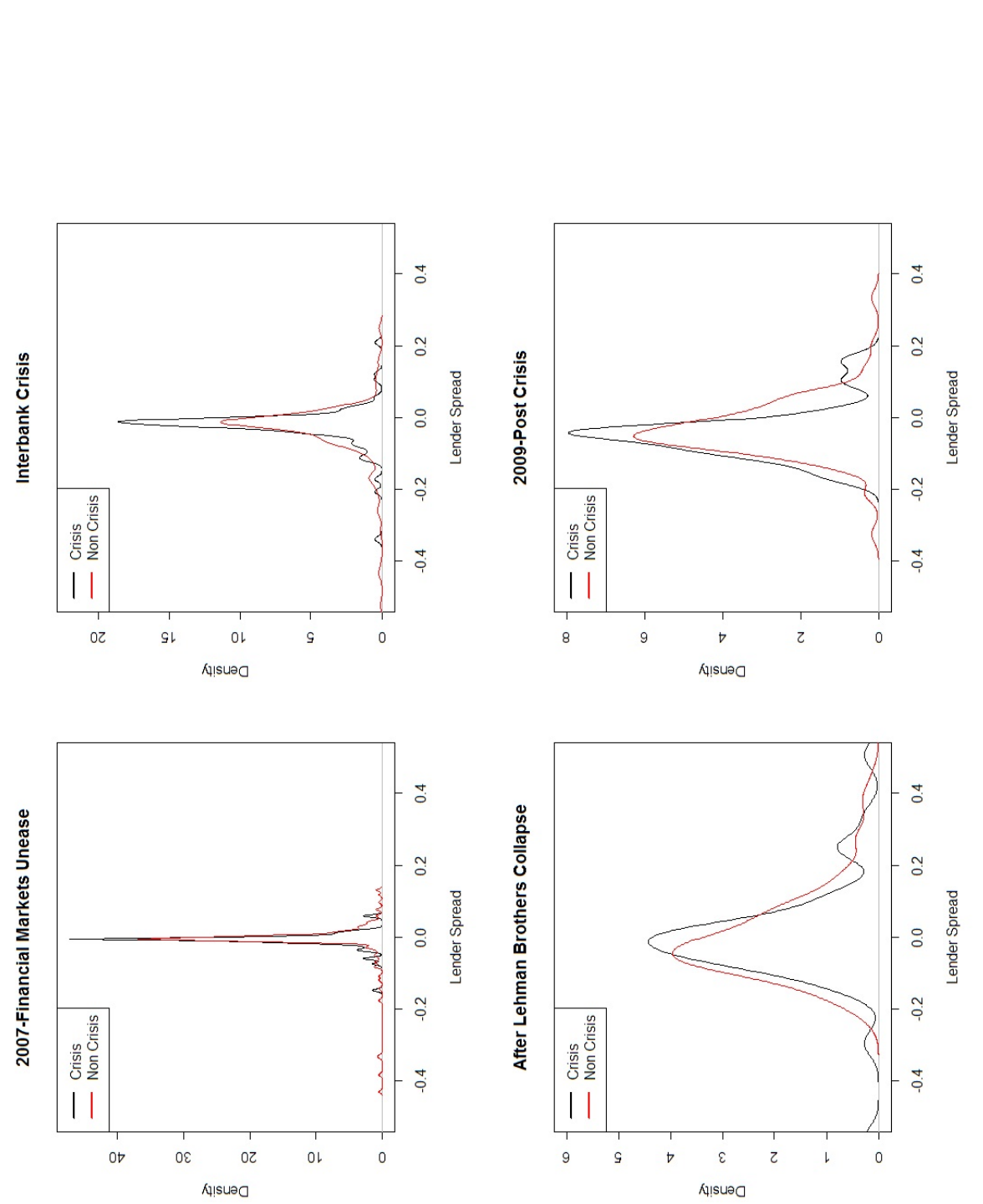

$\frac{\overline{0}}{\frac{0}{8}}$
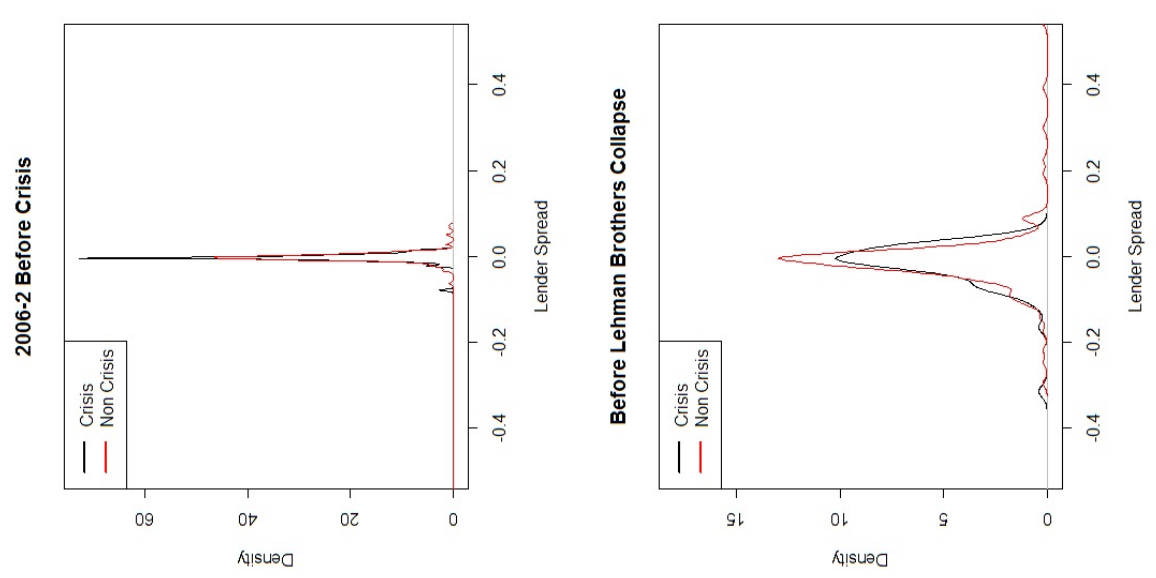

중

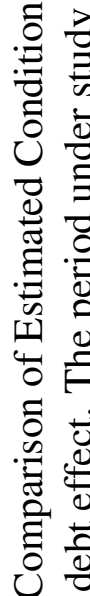

$\because$ ติ

히

齿 

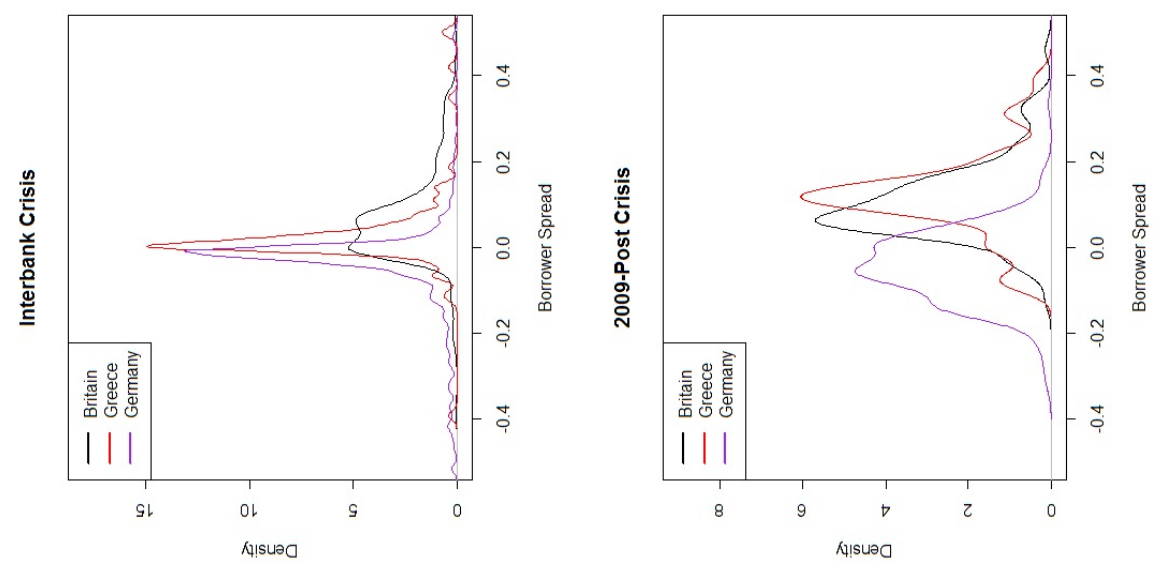

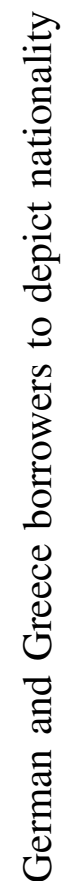
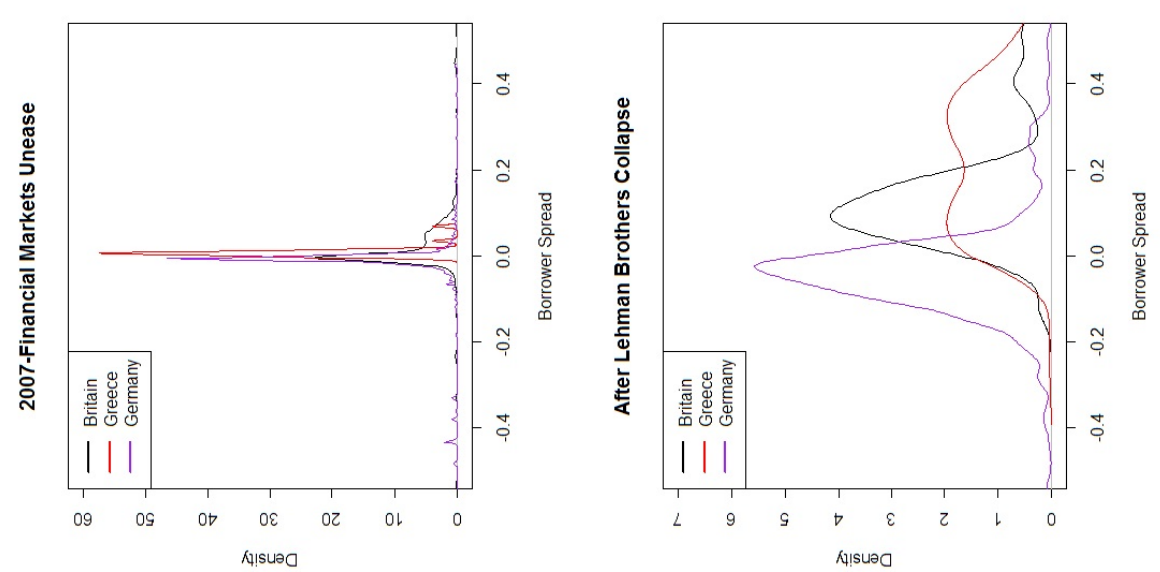

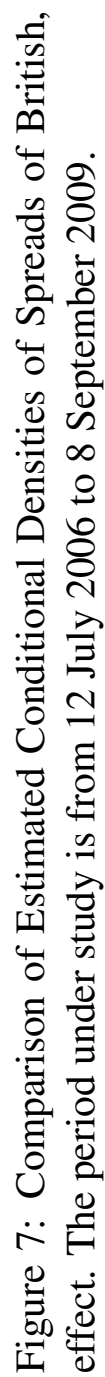

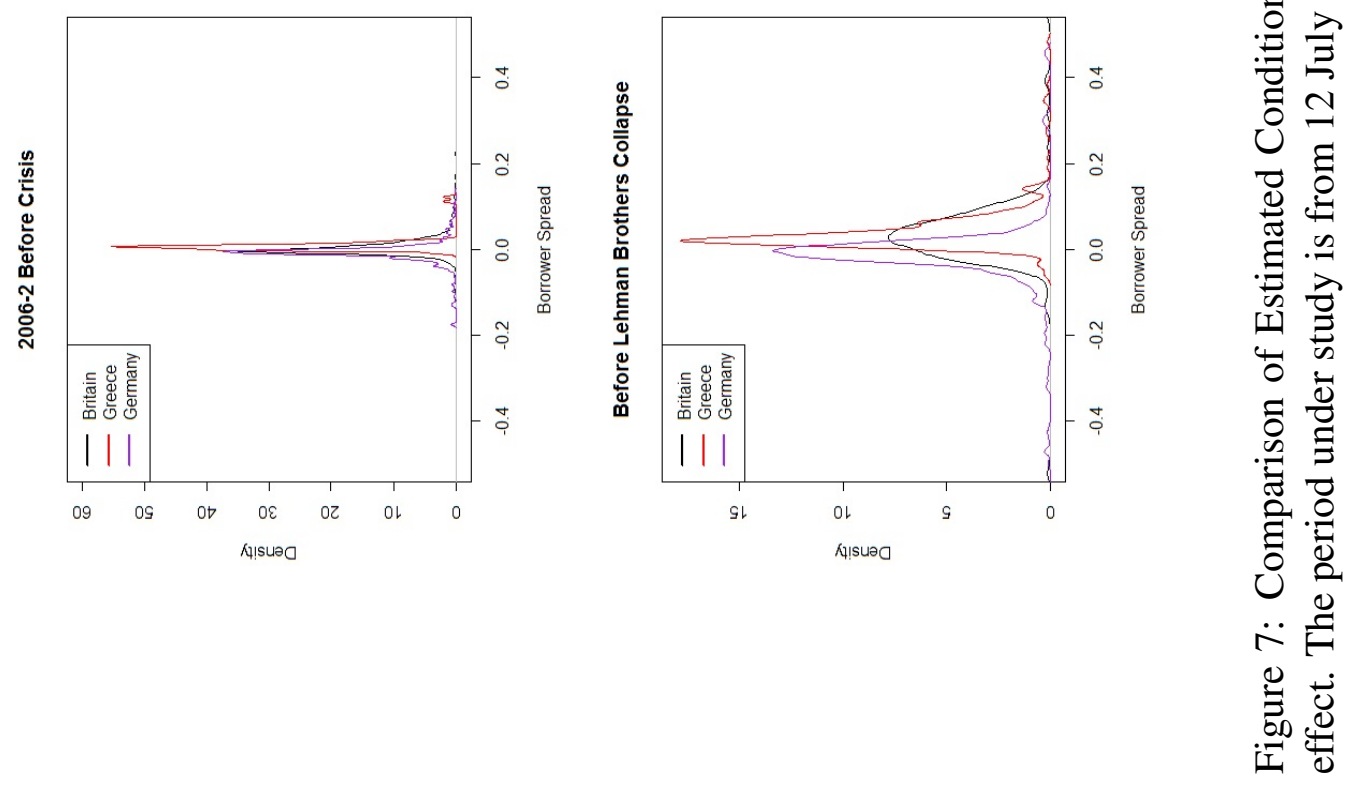



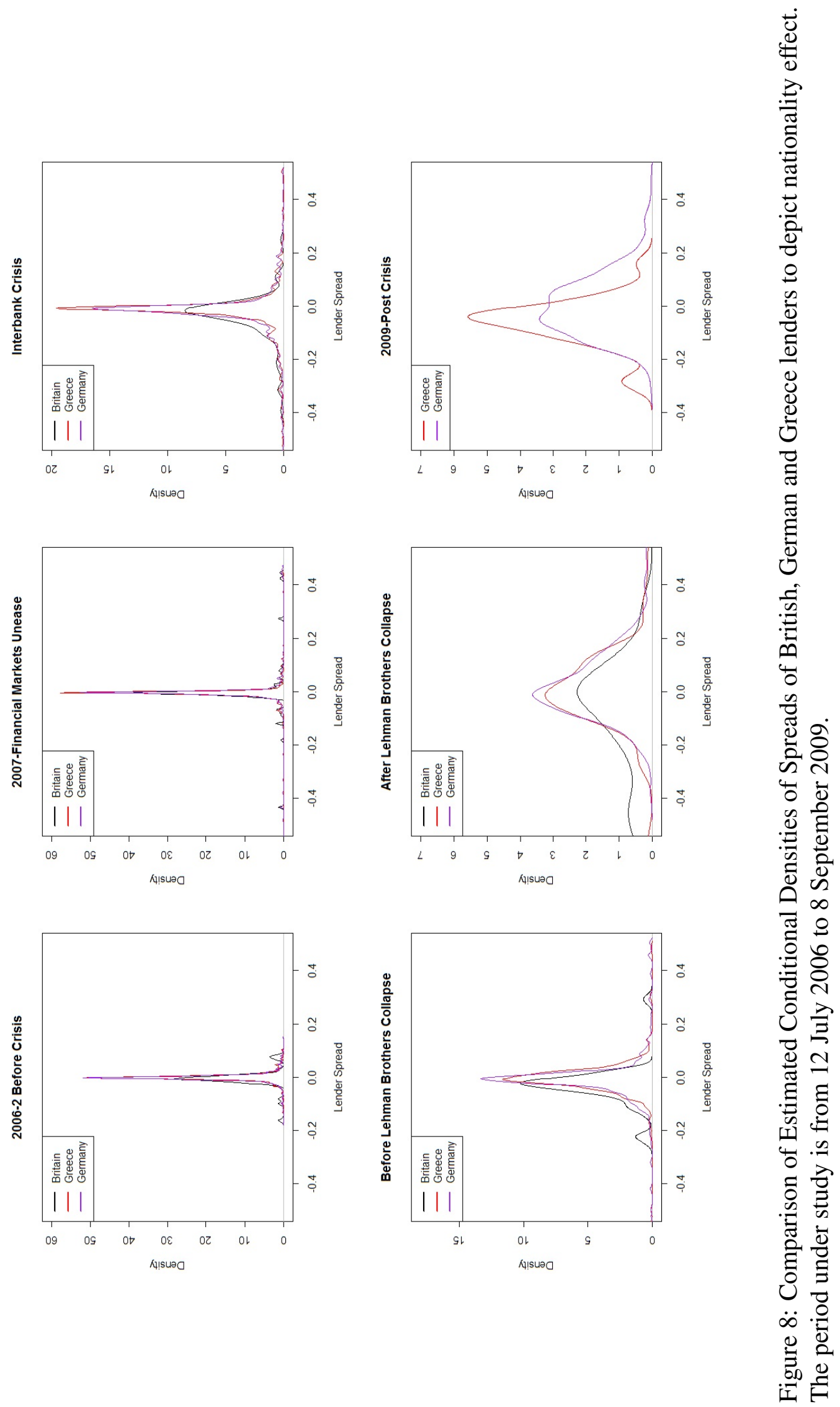

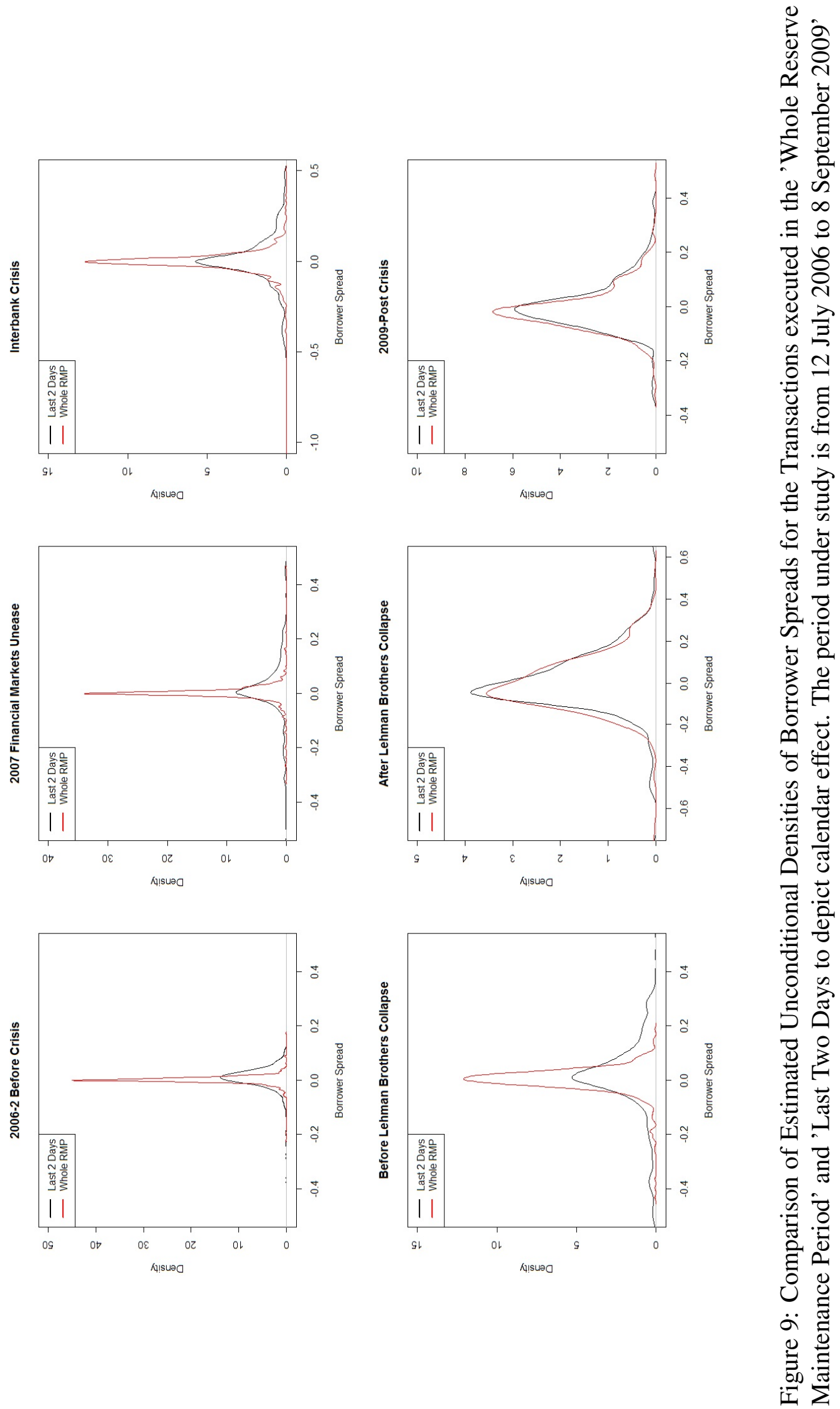

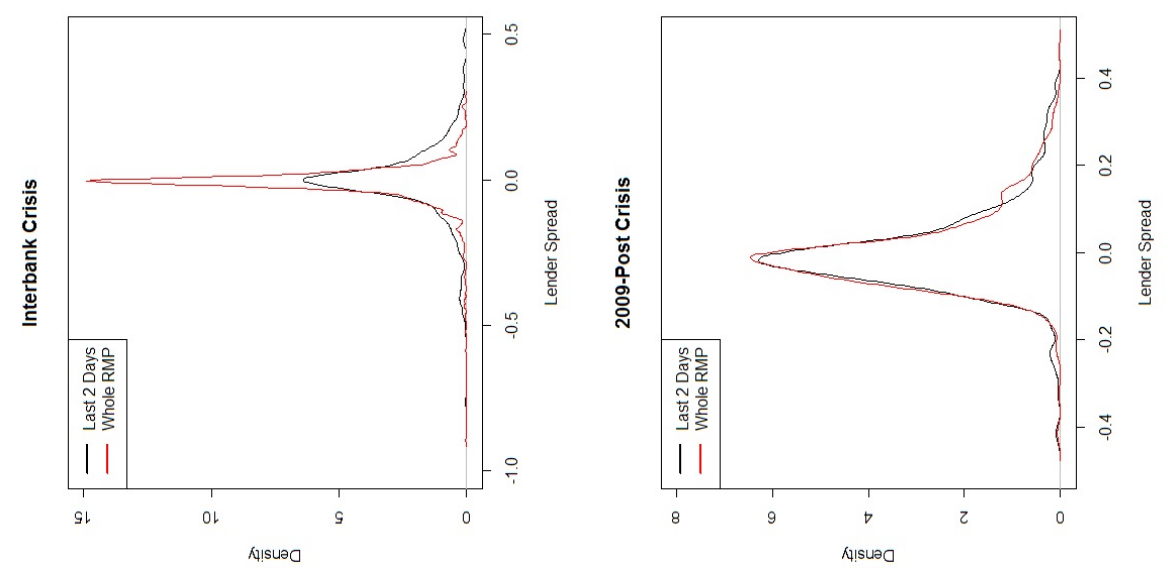

$\sum_{0}^{0}$
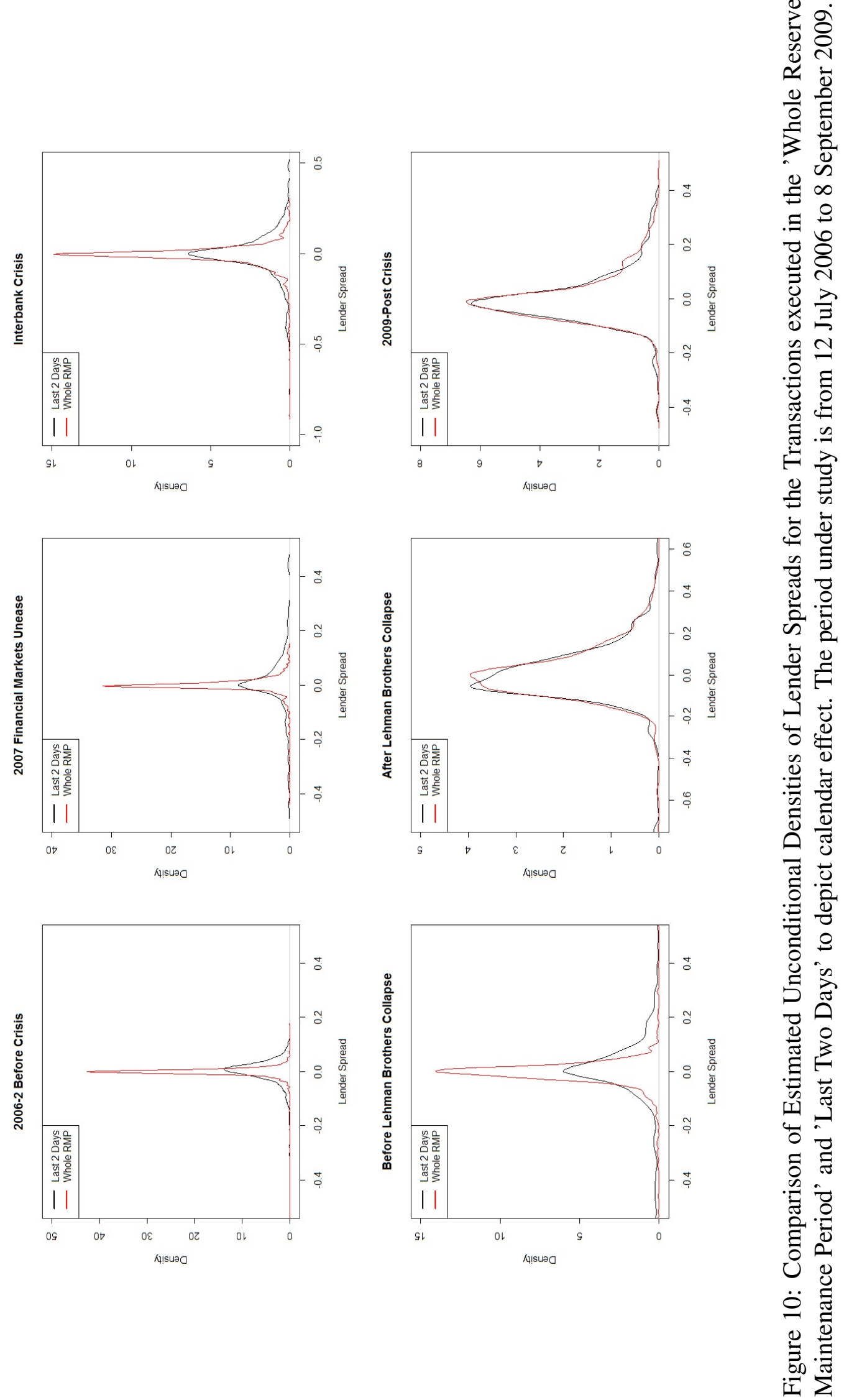

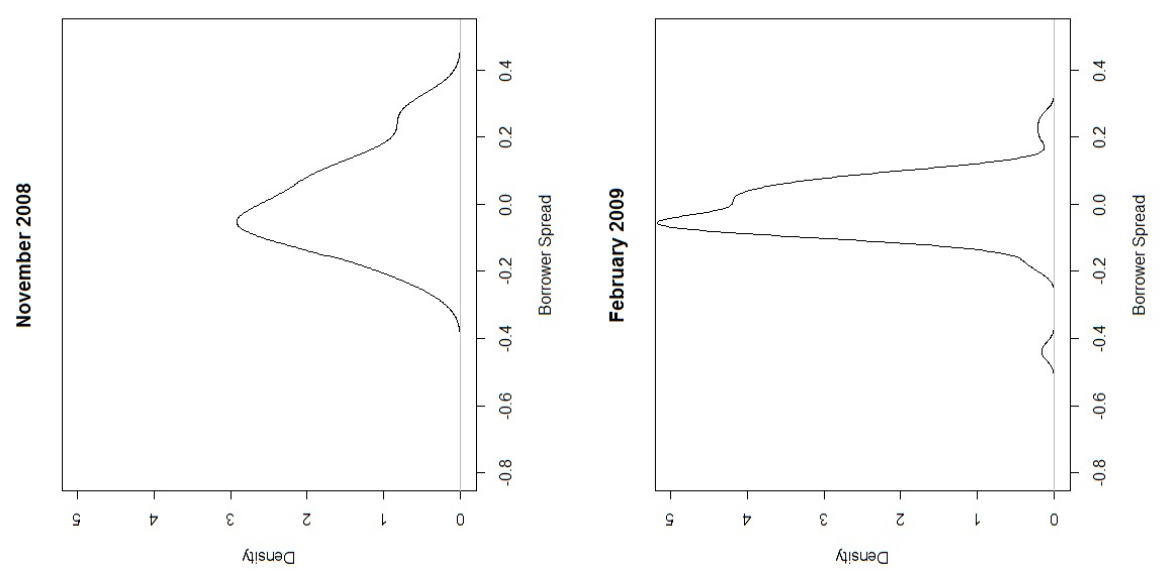

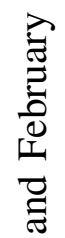
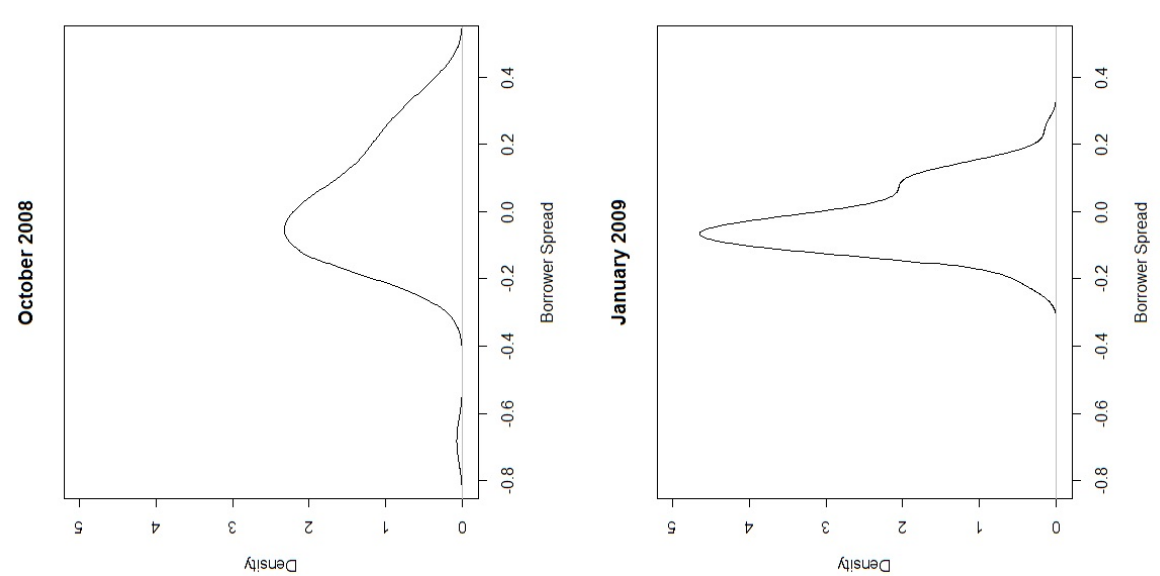

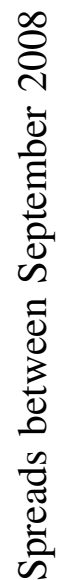

है
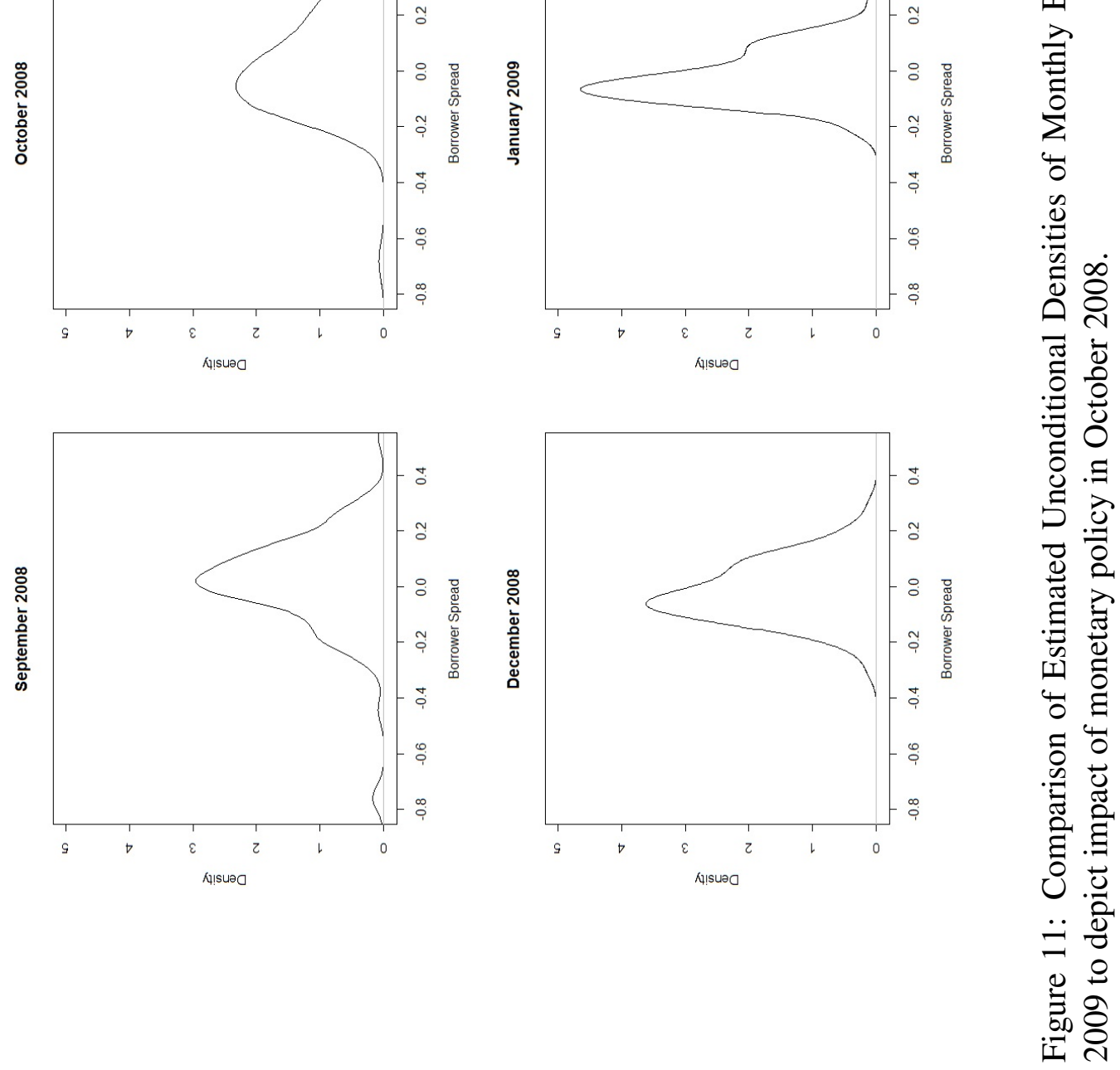


$$
9
$$



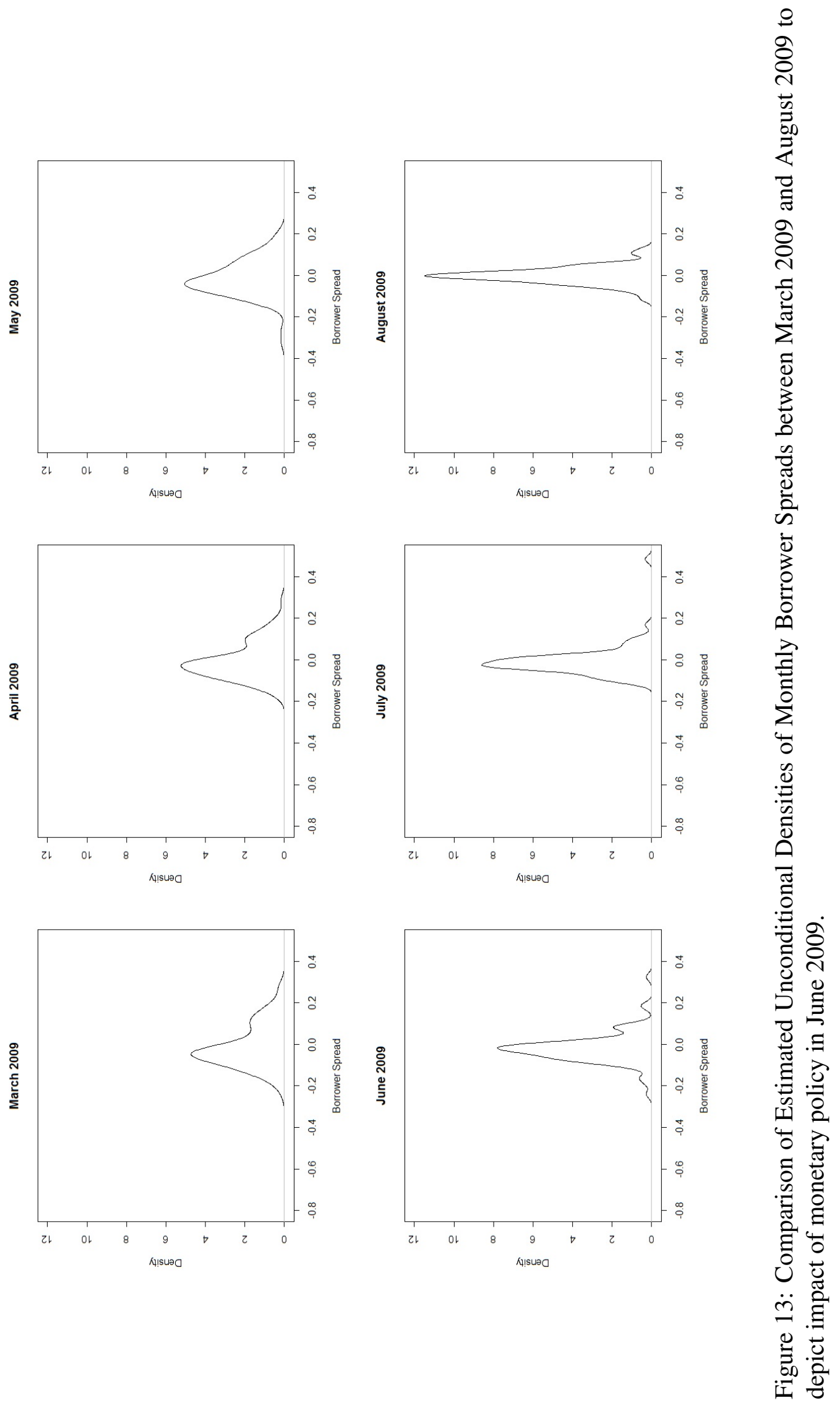

52 

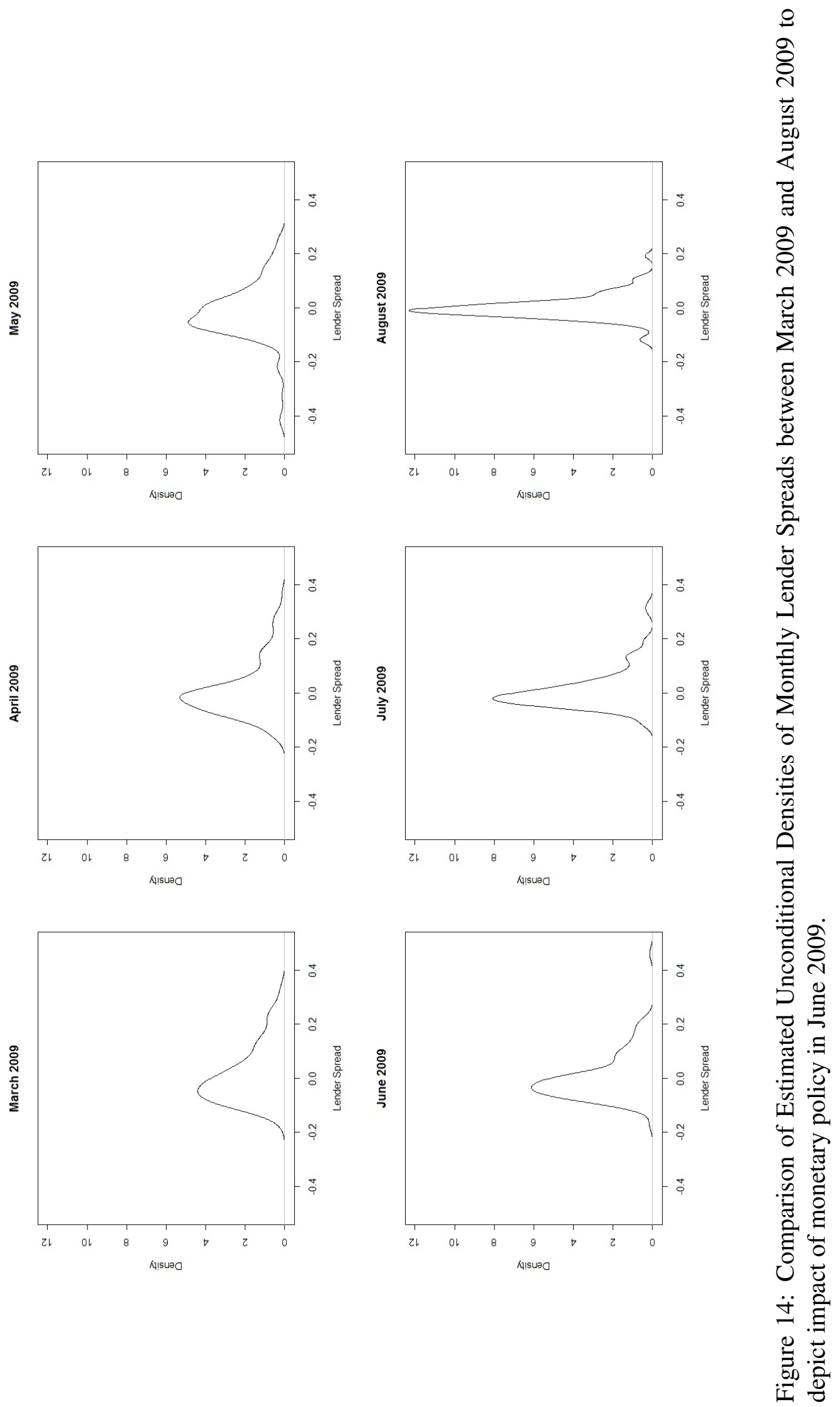

53 